\title{
Adaptive Robust Dynamic Surface Integral Sliding Mode Control for Quadrotor UAVs under Parametric Uncertainties and External Disturbances
}

\author{
Ye Zhang $\mathbb{D}^{1,2}$ Ning Xu, ${ }^{1,2}$ Guoqiang Zhu $\mathbb{D}^{1,2}$ Lingfang Sun, ${ }^{1,2}$ Shengxian Cao $\mathbb{D}^{1,2}$ \\ and Xiuyu Zhang ${ }^{1,2}$ \\ ${ }^{1}$ School of Automation Engineering, Northeast Electric Power University, Jilin, China \\ ${ }^{2}$ Jilin Province International Research Center of Precision Drive and Intelligent Control, Jilin, China \\ Correspondence should be addressed to Guoqiang Zhu; zhugqcn@gmail.com
}

Received 18 September 2020; Revised 4 November 2020; Accepted 16 November 2020; Published 27 November 2020

Academic Editor: Ning Wang

Copyright (c) 2020 Ye Zhang et al. This is an open access article distributed under the Creative Commons Attribution License, which permits unrestricted use, distribution, and reproduction in any medium, provided the original work is properly cited.

\begin{abstract}
A robust adaptive fuzzy nonlinear controller based on dynamic surface and integral sliding mode control strategy (ADSISMC) is proposed to realize trajectory tracking for a class of quadrotor UAVs. In this study, the composite factors including parametric uncertainties and external disturbances are added to controller design, which make it more realistic. The quadrotor model is divided into two subsystems of attitude and position that make the control design become feasible. The main contributions of the proposed ADSISMC strategy are as follows: (1) The combination of dynamic surface and integral sliding mode makes the system always in sliding stage by finding the appropriate initial position compared with the common sliding mode, and the complexity of explosion in backstepping method is eliminated. (2) By introducing the fuzzy system, the unknown functions and uncertainties can be approximated which significantly improves the robustness and the tracking performance. (3) The switching control strategy is utilized to compensate for the errors between estimated and ideal inputs; the tracking performance of the whole system has been significantly improved. The simulation results show the effectiveness of the proposed control method.
\end{abstract}

\section{Introduction}

As a newborn member of the small unmanned aerial vehicle (UVA) family, quadrotor has attracted much research interest due to its extensive utility in several important applicants, such as commercial photography, military surveillance, rescue mission, and agricultural investigation [1-4]. Compared with traditional unmanned fixed-wing flight vehicles and manned airplanes, the main advantages of the quadrotor lie in small size, low cost, stable hovering, vertical take-off and landing (VTOL), convenient portability, and versatile features [5]. However, trajectory tracking control of the quadrotor is a thorny problem because of its nonlinear, underactuated dynamics, and strong coupling $[6,7]$. Moreover, the quadrotor system is susceptible to external disturbances such as wind and nonlinear frictions. What is more, taking robustness of the trajectory tracking controller into consideration poses a bigger challenge [8].
In early quadrotor research stage, many studies used conventional linear control methods such as proportionalintegral-derivative (PID) $[9,10]$ and linear quadratic regulator (LQR) [11] to design the quadrotor controller in order to improve the simpleness and practicability. The linear control technology was developed to stabilize the quadrotor by neglecting the unimportant factors and linearizing the dynamic model. Therefore, it is poor and even not acceptable for the tracking accuracy and the robustness of the quadrotor. To overcome the drawbacks of the aforementioned linear control approaches, a large number of nonlinear control strategies, including backstepping control [12-15], sliding mode control (SMC) [16-20], and feedback linearization control, are utilized to improve the tracking performance of the flight control system.

Backstepping control method has received extensive attention, not only in the quadrotor control, but also in some mechanical systems. The main idea of backstepping technology 
is to select the appropriate state variable function as the recursive virtual control input. Once the final control input is received, the stability of the whole system is guaranteed. In [21-24], a backstepping controller has been designed to stabilize the attitude system of quadrotor. In [24], attitude control using hybrid backstepping methodology based on Frenet-Serret theory is studied in detail. The results show that the controller has good robustness under wind disturbance. To solve the problem of trajectory tracking, in [25], an adaptive controller combining parameter adaptive and backstepping control is designed. However, the obvious limitation of conventional backstepping design is the problem of "complexity of explosion" caused by the repeated differentiation of some nonlinear functions and the lack of robustness against uncertainties. To overcome this limitation of traditional backstepping control, dynamic surface control (DSC) is proposed as an effective alternative method [26-30]. In [27], a dynamic surface control method based on RBF neural network approximation is proposed for a class of nonlinear time-delay systems with state variables all measurable, which greatly simplifies the design process of the controller.

Moreover, to enhance the attitude performance robustness, disturbance observer (DOB), parameter estimation [31], and the approximation-based adaptive control are generally combined with DSC to handle external disturbances and parameterized uncertainties. For instance, a class of adaptive control methods using fuzzy logic systems or neural networks to approximate unknown functions in nonlinear systems have been proposed in [32-38]. In [33], a dynamic surface control-based adaptive fuzzy control method is proposed to overcome the "explosion of complexity" problem of classical backstepping. In [35], a robust dynamic surface controller based on extended state observer is presented for a quadrotor UAV subject to external disturbances and parametric uncertainties. In [36], both indirect and direct global neural controllers with the dynamic surface design are developed for the strict-feedback systems. The simulation results are presented to demonstrate the feasibility of the proposed global neural DSC design. A robot control scheme based on dynamic surface considering output error constraints, unknown dynamics, and bounded disturbance has been proposed in [39]; by introducing an improved virtual variable, the robustness of the control system was improved. However, the performance properties and robustness are not taken into account in these papers. As a commonly used nonlinear control method, the SMC is utilized as an effective method to design robust controllers for a specific class of nonlinear tracking problems in the presence of uncertain conditions [40-45]. Traditional SMC features the low sensitivity to the disturbances and parameter variations of the system [46-51]. In [50], a method based on second-order sliding mode control is used to avoid the chattering phenomenon for quadrotor UAVs. In [51], a robust backstepping sliding mode nonlinear controller for quadrotor UAVs is proposed to improve the robustness of the controller against model uncertainty and external disturbances. Compared with traditional sliding mode control method, the integral sliding mode (ISM) can guarantee that the system always meets the desired dynamic performance index during the whole arrival period which significantly improves the robustness of the control system.

Motivated by the aforementioned observations, a new control methodology combined with dynamic surface and ISMC is proposed for the quadrotor trajectory tracking problem under parametric uncertainties and external disturbances. The main contributions of this paper are summarized as follows: (1) By fusing the technique of DSC and the integral SMC, a new integral sliding mode robust dynamic surface trajectory tracking controller is designed, which eliminates the "explosion of complexity" in the backstepping and improves the robustness of the whole system. (2) The FLSs are introduced to approach the ideal control law. And the estimations of the weight vector norm are utilized in the FLSs to significantly reduce the number of online estimation parameters. Therefore, the amount of calculation is obviously reduced, and the structure of the proposed controller is simplified. (3) The adaptive switching control is introduced to compensate the error between the real control law and the ideal control law, and the tracking performance of the whole system has been significantly improved.

The rest of this paper is organized as follows. The modeling of a quadrotor and some preliminaries are introduced in Section 2. The control algorithms are introduced in Section 3. Section 4 gives the stability analysis of the control system. Extensive simulations under different operating scenarios are given in Section 5. This paper ends with the conclusions in Section 6.

\section{Problem Formulation and Preliminaries}

2.1. The Mathematical Model of UAV. The quadrotor UAV is an underactuated system because it has six degrees of freedom, but only four actual inputs $[1,2]$. In this paper, the quadrotor UAV with four rotors is shown in Figure 1. The equations of the dynamic quadrotor UAV are basically a rotating rigid body with six degrees of freedom $[4,5]$ which are usually derived by Newton-Euler formulas [8-10].

Define $\xi=[\phi, \theta, \varphi]^{T}$ and $\omega_{b}=[p, q, r]^{T}$, with $\varphi, \theta$, and $\phi$ being the angle of roll, pitch, and yaw with respect to the inertia frame. $p, q$, and $r$ are the angular velocity of roll, pitch, and yaw with respect to the body-fixed frame. The rotation matrix from the rigid frame to the inertia frame can be expressed as

$$
R_{t}=\left(\begin{array}{ccc}
C_{\varphi} C_{\theta} & C_{\varphi} S_{\theta} S_{\phi}-S_{\varphi} C_{\phi} & C_{\varphi} S_{\theta} C_{\phi}+S_{\varphi} S_{\phi} \\
S_{\varphi} C_{\theta} & S_{\varphi} S_{\theta} S_{\phi}+C_{\varphi} C_{\phi} & S_{\varphi} S_{\theta} C_{\phi}-C_{\varphi} S_{\phi} \\
-S_{\theta} & C_{\theta} S_{\phi} & C_{\theta} C_{\phi}
\end{array}\right),
$$

where $S_{(\cdot)}$ and $C_{(\cdot)}$ denote $\sin (\cdot)$ and $\cos (\cdot)$, respectively. According to the rotation matrix $R_{t}$, the relationship between $\dot{\xi}$ and $\omega_{b}$ can be described as

$$
\omega_{b}=R_{r} \dot{\xi}=\left(\begin{array}{ccc}
1 & 0 & -S_{\theta} \\
0 & C_{\phi} & C_{\theta} S_{\phi} \\
0 & -S_{\phi} & C_{\theta} C_{\phi}
\end{array}\right)\left(\begin{array}{c}
\dot{\phi} \\
\dot{\theta} \\
\dot{\varphi}
\end{array}\right) \text {. }
$$




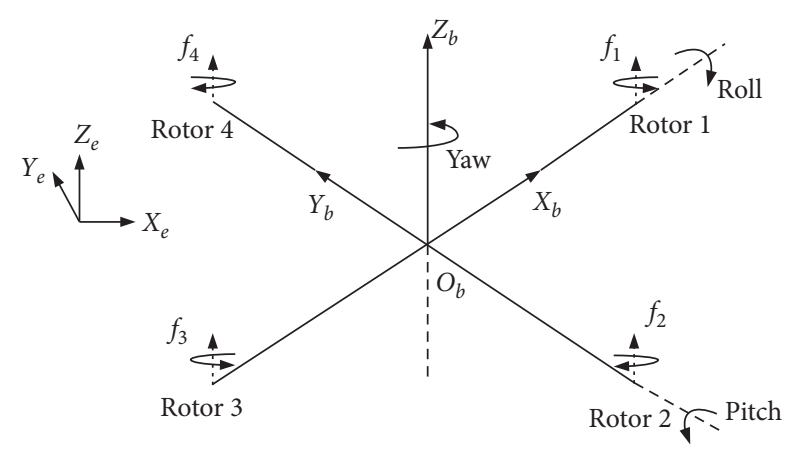

FIGURE 1: Schematic of the quadrotor UAV.

Define $M_{b}$ as the torque provided by the rotors with respect to the body-fixed frame, and it is presented as follows:

$$
M_{b}=\left(\begin{array}{c}
M_{b x} \\
M_{b y} \\
M_{b z}
\end{array}\right)=\left(\begin{array}{c}
l_{k}\left(\Omega_{4}^{2}-\Omega_{2}^{2}\right) \\
l_{k}\left(\Omega_{3}^{2}-\Omega_{1}^{2}\right) \\
l\left(\Omega_{4}^{2}+\Omega_{2}^{2}-\Omega_{1}^{2}-\Omega_{3}^{2}\right)
\end{array}\right),
$$

where $\Omega_{i}$ denotes the rotary speed of the front, right, rear, and left rotors, respectively; $l_{k}$ is the distance between a rotor and the center of mass of the quadrotor; $k$ is the drag force coefficient; and $l$ is the reverse moment coefficient. Using the Newton-Euler equation, the rotational dynamic equation of the quadrotor is obtained as follows:

$$
M_{b}=J_{b} \dot{\omega}_{b}+\omega_{b} \times J_{b} \omega_{b}+M_{g}+M_{d},
$$

where $J_{b}=\operatorname{diag}\left(J_{x}, J_{y}, J_{z}\right)$ is a symmetric positive definite constant matrix with $J_{x}, J_{y}$, and $J_{z}$ being the rotary inertia with respect to the $O_{b} X_{b}, O_{b} Y_{b}$, and $O_{b} Z_{b}$ axes, respectively; the notation $\times$ denotes cross multiplication; $M_{q}$ and $M_{d}$ are the resultant torques due to the resultant of aerodynamic frictions torque and the gyroscopic effects. They are given as

$$
M_{g}=\sum_{i=1}^{4} \omega_{b} \times J_{r}\left[0,0,(-1)^{i+1} \Omega_{i}\right]^{T}, M_{d}=\operatorname{diag}\left(d_{\phi}, d_{\theta}, d_{\varphi}\right) \dot{\xi},
$$

where $J_{r}$ denotes the moment of inertia of each rotor; $d_{\phi}, d_{\theta}$, and $d_{\varphi}$ are the corresponding aerodynamic drag coefficients. According to (4), the following equation can be obtained:

$$
\dot{\omega}_{b}=J_{b}^{-1}\left[M_{b}-M_{g}-M_{d}-\omega_{b} \times\left(J_{b} \omega_{b}\right)\right] .
$$

Furthermore, with the help of approximation of Euler angles at equilibrium point, the following dynamic equations can be obtained:

$$
\left\{\begin{array}{l}
\ddot{\phi}=\frac{\left[\dot{\theta} \dot{\varphi}\left(J_{y}-J_{z}\right)-J_{r} \dot{\theta} \omega-d_{\phi} \dot{\phi}+M_{b x}\right]}{J_{x}}, \\
\ddot{\theta}=\frac{\left[\dot{\phi} \dot{\varphi}\left(J_{z}-J_{x}\right)-J_{r} \dot{\phi} \oplus-d_{\theta} \dot{\theta}+M_{b y}\right]}{J_{y}}, \\
\ddot{\varphi}=\frac{\left[\dot{\phi} \dot{\theta}\left(J_{x}-J_{y}\right)-d_{\dot{\varphi}} \dot{\varphi}+M_{b z}\right]}{J_{z}},
\end{array}\right.
$$

where $\Phi=\Omega_{4}+\Omega_{3}-\Omega_{2}-\Omega_{1}$ can be got easily online. It should be noted that to make the roll and pitch angles physically meaningful, they are both limited to $(-\pi / 2, \pi / 2)$. In particular, the yaw angle is also limited to $(-\pi / 2, \pi / 2)$ in this study, while $P=[x, y, z]^{T} \in R^{3}$ is the position with respect to the inertial frame. The translational dynamic equations of the quadrotor are given as

$$
m \ddot{P}=R_{t} \cdot F+\left[\begin{array}{c}
0 \\
0 \\
-m g
\end{array}\right]-\left[\begin{array}{l}
d_{x} \dot{x} \\
d_{y} \dot{y} \\
d_{z} \dot{z}
\end{array}\right],
$$

where $d_{x}, d_{y}$, and $d_{z}$ are the air drag coefficients which are added in (8) to model the drag force caused by translational motions; $F$ is the lift force generated by rotors with respect to the body-fixed frame.

$$
F=\left[\begin{array}{c}
0 \\
0 \\
k\left(\Omega_{1}^{2}+\Omega_{2}^{2}+\Omega_{3}^{2}+\Omega_{4}^{2}\right)
\end{array}\right] .
$$

By combining (7) and (8), a compact affine nonlinear equation of the quadrotor $\mathrm{UAV}$ is given as

$$
\dot{X}=f(X)+g(X) U,
$$

where $X=[x, \dot{x}, y, \dot{y}, z, \dot{z}, \phi, \dot{\phi}, \theta, \dot{\theta}, \varphi, \dot{\varphi}]^{T} \in R^{12}$ is the state variable; $f(X)$ and $g(X)$ are smooth functions on $X$. Equation (10) is expended as follows:

$$
\left\{\begin{array}{l}
\dot{x}_{1}=x_{2}, \\
\dot{x}_{2}=\left(C_{x 7} S_{x 9} C_{x 11}+S_{x 7} S_{x 11}\right) U_{1}-a_{1} x_{2}+d_{1}, \\
\dot{x}_{3}=x_{4}, \\
\dot{x}_{4}=\left(C_{x 7} S_{x 9} S_{x 11}-S_{x 7} S_{x 11}\right) U_{1}-a_{2} x_{4}+d_{2}, \\
\dot{x}_{5}=x_{6} \\
\dot{x}_{6}=\left(C_{x 7} C_{x 9}\right) U_{1}-g-a_{3} x_{6}+d_{3}, \\
\dot{x}_{7}=x_{8}, \\
\dot{x}_{8}=a_{4} x_{10} x_{12}+a_{5} @ x_{10}-a_{6} x_{8}+U_{2}+d_{4}, \\
\dot{x}_{9}=x_{10}, \\
\dot{x}_{10}=a_{7} x_{8} x_{12}+a_{8} \bowtie x_{8}-a_{9} x_{10}+U_{3}+d_{5}, \\
\dot{x}_{11}=x_{12}, \\
\dot{x}_{12}=a_{10} x_{8} x_{10}-a_{11} x_{12}+U_{4}+d_{6},
\end{array}\right.
$$

where $U_{i}, i=1,2,3,4$ represents the control inputs defined as follows:

$$
\begin{aligned}
& U_{1}=\frac{l_{k}\left(\Omega_{1}^{2}+\Omega_{2}^{2}+\Omega_{3}^{2}+\Omega_{4}^{2}\right)}{m}, \\
& U_{2}=\frac{l_{k}\left(\Omega_{4}^{2}-\Omega_{2}^{2}\right)}{J x}, \\
& U_{3}=\frac{l_{k}\left(\Omega_{3}^{2}-\Omega_{1}^{2}\right)}{J y}, \\
& U_{4}=\frac{l_{k}\left(\Omega_{4}^{2}+\Omega_{2}^{2}-\Omega_{3}^{2}-\Omega_{1}^{2}\right)}{J z},
\end{aligned}
$$




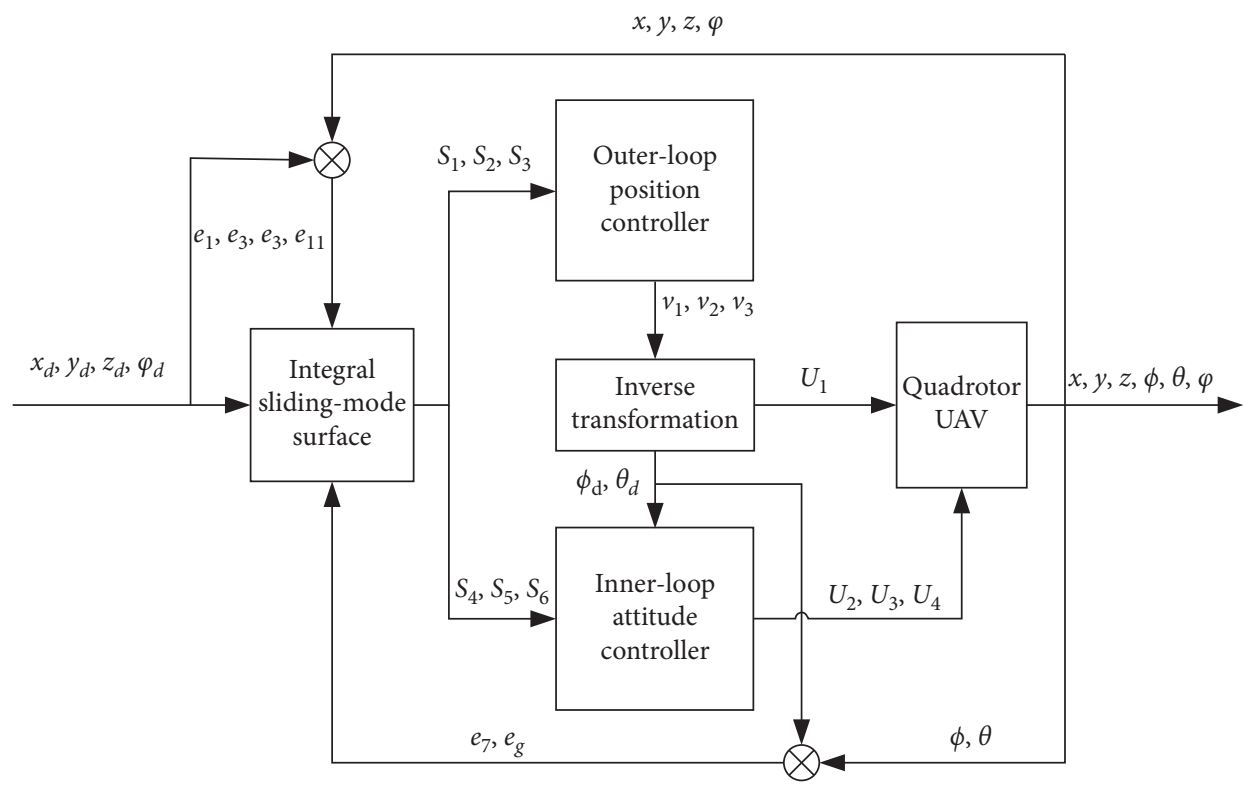

FIGURE 2: Schematic diagram of the proposed control scheme.

and $a_{i}, i=1,2, \ldots, 11$, are the normalized parameters defined as follows:

$$
\begin{aligned}
& a_{1}=\frac{d_{x}}{m}, \\
& a_{2}=\frac{d_{y}}{m}, \\
& a_{3}=\frac{d_{z}}{m}, \\
& a_{4}=\frac{J_{y}-J_{z}}{J_{x}}, \\
& a_{5}=\frac{J_{r}}{J_{x}} \\
& a_{6}=\frac{d_{\phi}}{J_{x}}, \\
& a_{7}=\frac{J_{z}-J_{x}}{J_{y}}, \\
& a_{10}=\frac{J_{x}-J_{y}}{J_{z}}, \\
& a_{8}=\frac{J_{r}}{J_{y}}, \\
& a_{y}
\end{aligned}
$$

2.2. Fuzzy Logic Systems (FLSs). In this study, the Fuzzy Logic Systems (FLSs) are introduced to approximate the continuous unknown functions on a given compact set. The FLSs consist of three main parts: fuzzy rule base, fuzzification, and defuzzification operators. The form of the fuzzy rules of the fuzzy controller is

Rule $l$ : If $x_{1}$ is $F_{1}^{l}$ and $x_{2}$ is $F_{2}^{l}$ and ... and $x_{n}$ is $F_{n}^{l}$.

Then $y$ is $G^{l}, l=1,2, \ldots, N$. where $x(t)=\left[x_{1}, x_{2}, \ldots, x_{n}\right]^{T}$ and $y$ are the input and output of the whole fuzzy system, respectively. And $N$ is the number of the rules. The fuzzy basis functions forms are defined as

$$
y(x)=\frac{\sum_{l=1}^{N} \bar{y}^{l}\left(\prod_{i=1}^{n} \mu_{F_{i}^{l}}\left(x_{i}\right)\right)}{\sum_{l=1}^{N}\left(\prod_{i=1}^{n} \mu_{F_{i}^{l}}\left(x_{i}\right)\right)},
$$

where $\bar{y}^{l}=\max _{y \in R} \mu_{G^{l}}(y)$.

$$
\xi_{l}(x)=\frac{\prod_{i=1}^{n} \mu_{F_{i}^{l}}\left(x_{i}\right)}{\sum_{l=1}^{N}\left(\prod_{i=1}^{n} \mu_{F_{i}^{l}}\left(x_{i}\right)\right)} .
$$

Denoting $\alpha^{T}=\left[\bar{y}_{1}, \bar{y}_{2}, \ldots, \bar{y}_{N}\right]=\left[\alpha_{1}, \alpha_{2}, \ldots, \alpha_{N}\right]$ and $\xi(x)=\left[\xi_{1}(x), \xi_{2}(x), \ldots, \xi_{N}(x)\right]^{T}$, then equation of the fuzzy system can be rewritten as

$$
y(x)=\alpha^{T} \xi(x)
$$

Lemma 1. For a continuous nonlinear function $f(x)$ in a compact set $\Omega_{x}$, it can be effectively approximated by FLSs with any small approximated error $\varepsilon>0 . f(x)$ can be expressed as follows:

$$
\sup _{x \in \Omega_{x}}\left|f(x)-\alpha^{T} \xi(x)\right|<\varepsilon
$$


Table 1: Position control algorithm of UAVs.

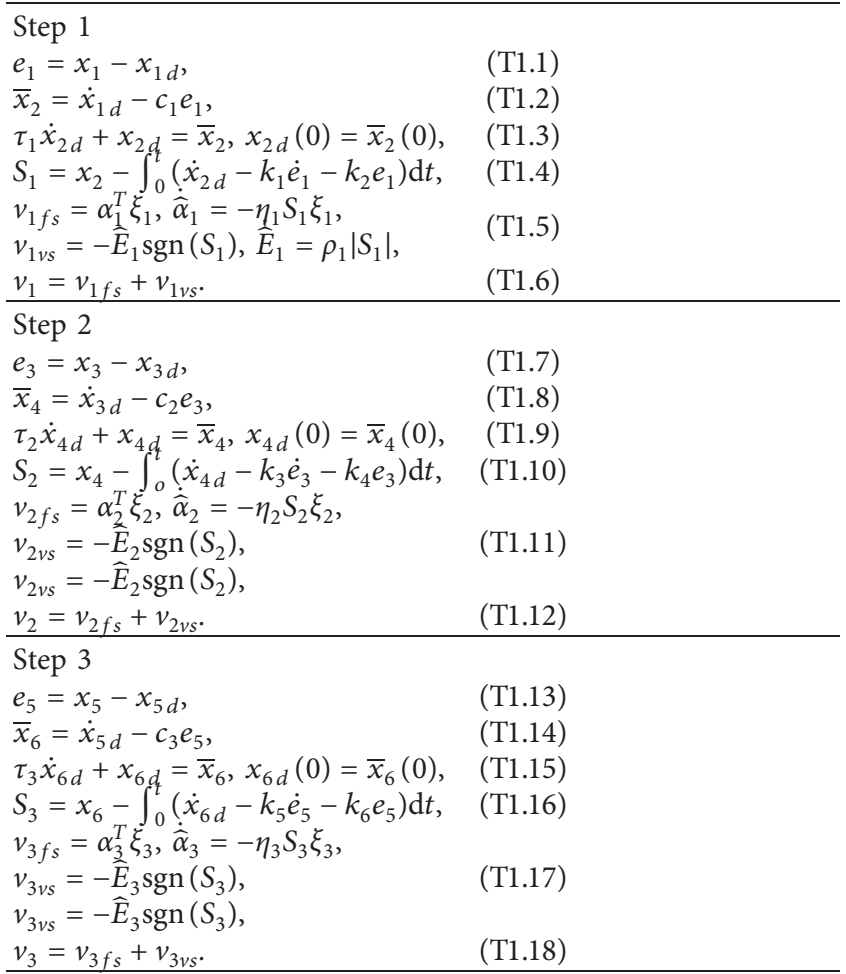

if $x \in \Omega_{x}$, then the smooth nonlinear function $f(x)$ can be expressed as

$$
f(x)=\alpha^{* T} \xi(x)+\varepsilon(x),
$$

where $\alpha^{*}$ is the optimal fuzzy parameter vector, $\varepsilon(x)$ is the approximation error satisfies $\|\varepsilon(x)\| \leq \bar{\varepsilon} \bar{\varepsilon}>0$.

\section{DSISMC Controller Design Procedure}

In this section, the design process of dynamic surface integral sliding mode controller is proposed and the control system block diagram is shown in Figure 2. The controller design process is divided into two parts: the position tracking controller design and attitude tracking controller design, as shown in Tables 1 and 2, respectively. To make the presentation clear, the specific controller design process is given in Appendix A.

In Table $1, e_{i},(i=1,2,3)$ are the tracking error, and $\bar{x}_{i},(i=2,4,6)$ are the virtual laws. The low-pass first-order filters (T1.3), (T1.9), and (T1.15) are presented in each step to get a new variable $x_{\mathrm{id}},(i=2,4,6)$ with the time constants $\tau_{i},(i=1,2,3)$. The integral sliding mode surfaces $S_{i},(i=$ $1,2,3)$ are selected in each step which improves the robustness of the system against disturbances and parameters uncertainties. Due to the existence of unknown functions and parameters, the fuzzy system $v_{i f s},(i=1,2,3)$ are utilized to approximate $v_{i},(i=1,2,3)$ with $\alpha_{i},(i=1,2,3)$ and $\xi_{i},(i=1,2,3)$ being the adjustable parameters and fuzzy basis vectors, respectively. Then, the switching control law $v_{i v s},(i=1,2,3)$ are introduced to compensate the error of $v_{i},(i=1,2,3)$ and the ideal input. $E_{i},(i=1,2,3)$ are the switching gain, $c_{i}, \eta_{i}, \rho_{i},(i=1,2,3)$, and $k_{i},(i=1,2, \ldots, 6)$
TABLE 2: Attitude control algorithm of UAVs.

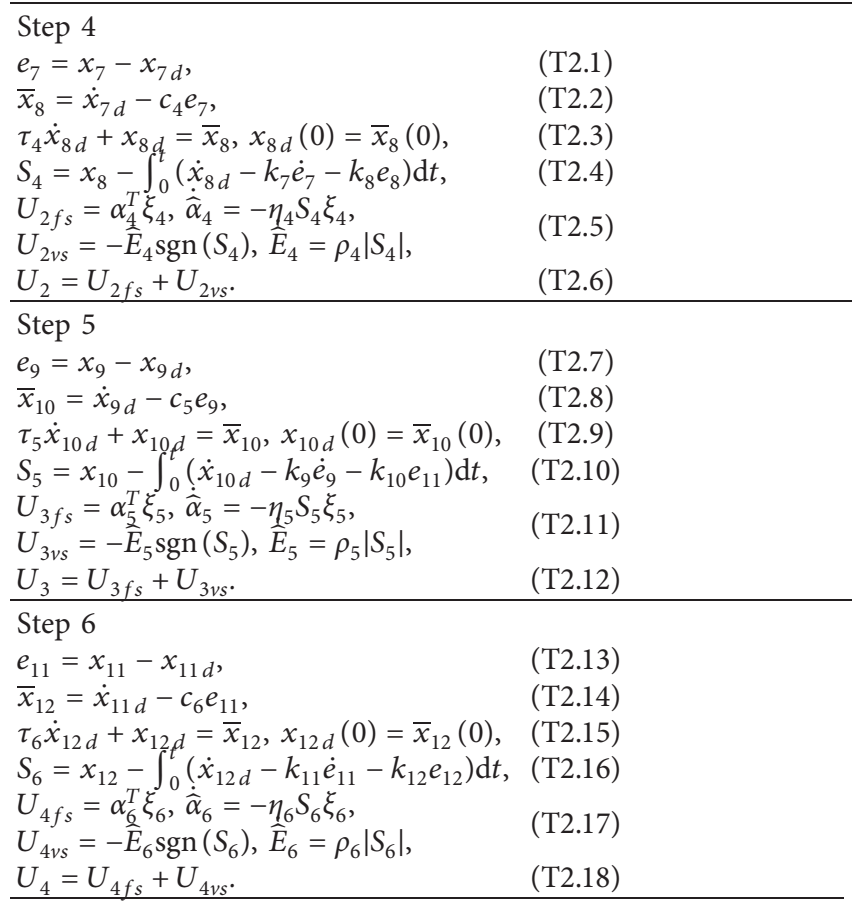

TABLE 3: Quadrotor parameters.

\begin{tabular}{lccccc}
\hline Symbol & Case1 & Case 2 & Case 3 & Case 4 & Units \\
\hline$m$ & 2 & 2 & 2 & 2 & $\mathrm{~kg}$ \\
$l$ & 0.2 & 0.2 & 0.2 & 0.2 & $\mathrm{~m}$ \\
$\kappa$ & 2.98 & 2.98 & 2.98 & 2.98 & $10^{-6} \mathrm{~N} \cdot \mathrm{s}^{2} \cdot \mathrm{rad}^{-2}$ \\
$\tau$ & 1.14 & 1.14 & 1.14 & 1.14 & $10^{-7} \mathrm{~N} \cdot \mathrm{s}^{2} \cdot \mathrm{rad}^{-2}$ \\
$d_{\phi}$ & 1.2 & 1.2 & 1.2 & 1.2 & $10^{-2} \mathrm{~N} \cdot \mathrm{s} \cdot \mathrm{rad}^{-1}$ \\
$d_{\theta} d_{\phi}$ & 1.2 & 1.2 & 1.2 & 1.2 & $10^{-2} \mathrm{~N} \cdot \mathrm{s} \cdot \mathrm{rad}^{-1}$ \\
$J_{x}$ & 1.25 & 1.25 & 1.25 & 1.25 & $\mathrm{~N} \cdot \mathrm{s}^{2} \cdot \mathrm{rad}^{-1}$ \\
$J_{y}$ & 1.25 & 1.25 & 1.25 & 1.25 & $\mathrm{~N} \cdot \mathrm{s}^{2} \cdot \mathrm{rad}^{-1}$ \\
$J_{z}$ & 2.50 & 2.88 & 3.25 & 3.75 & $\mathrm{~N} \cdot \mathrm{s}^{2} \cdot \mathrm{rad}^{-1}$ \\
\hline
\end{tabular}

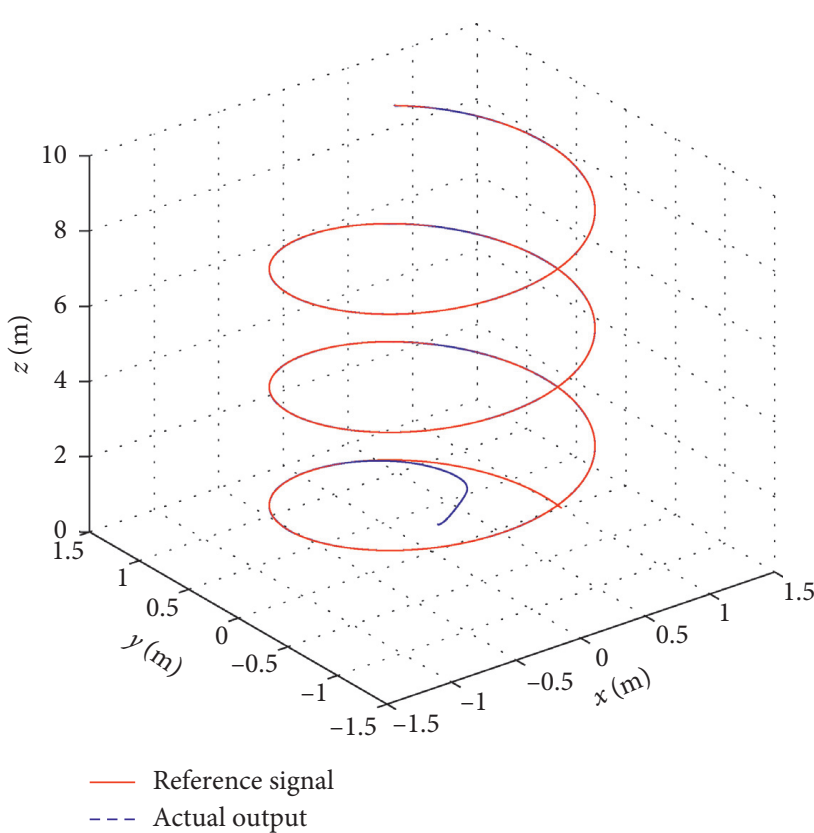

Figure 3: Space diagram of position in normal case. 

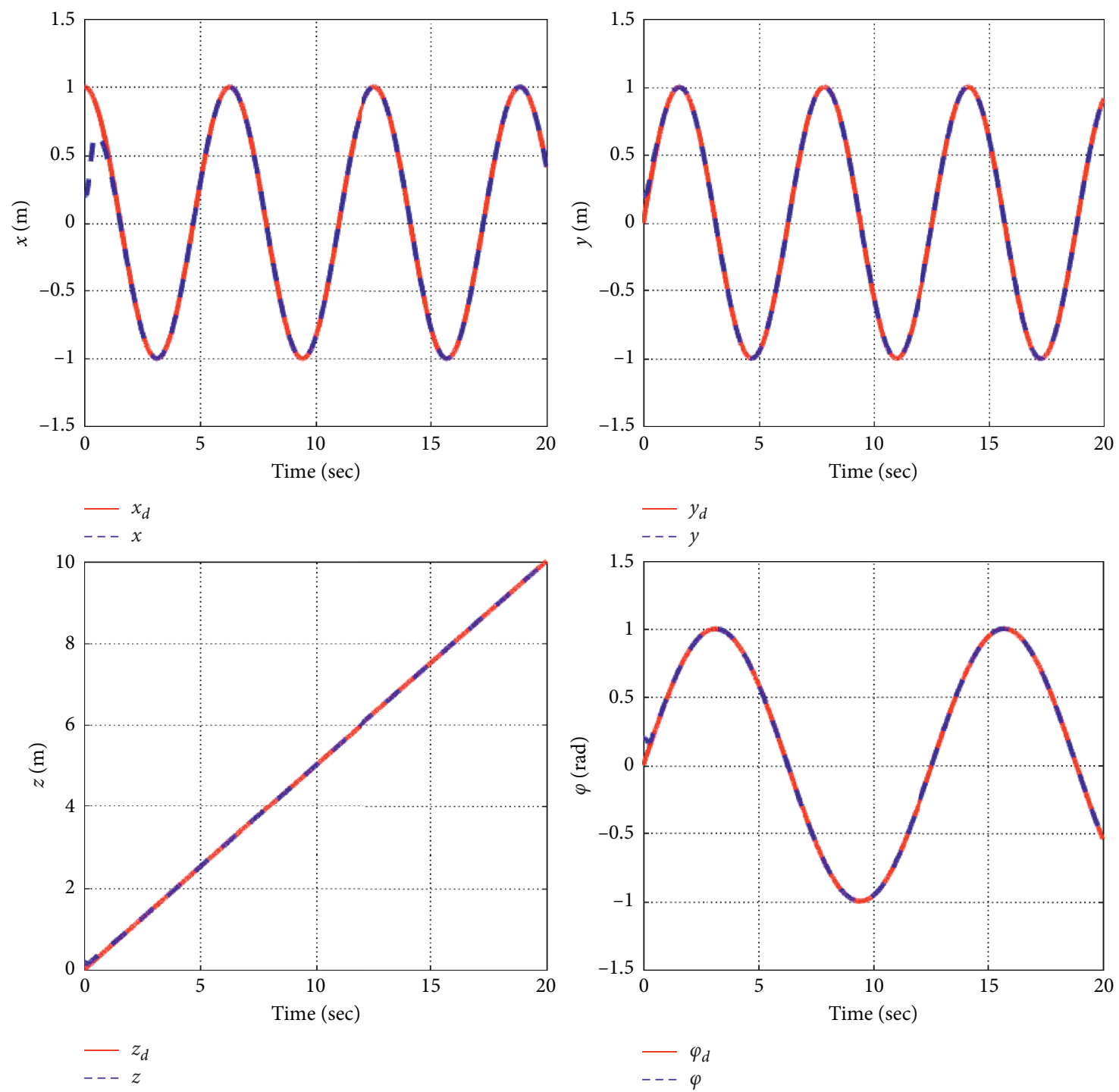

FIGURE 4: Reference signal and the actual outputs.

are positive constant. It is worth noting that $v=\left[v_{1}, v_{2}, v_{3}\right]^{T}$ is a group of virtual control laws and they can be given by $v_{1}=\left(C_{x 7} S_{x 9} C_{x 11}+S_{x 7} S_{x 11}\right) U_{1}, \quad v_{2}=\left(C_{x 7} S_{x 9} S_{x 11}-S_{x 7} S_{x 11}\right)$ $U_{1}, v_{3}=\left(C_{x 7} C_{x 9}\right) U_{1}$.

In Table 2, $e_{i},(i=4,5,6)$ are the tracking error, and $\bar{x}_{i},(i=8,10,12)$ are the virtual laws. The low-pass firstorder filters (T2.3), (T2.9), and (T2.15) are presented in each step to get a new variable $x_{\text {id }},(i=8,10,12)$ with the time constants $\tau_{i},(i=4,5,6)$. The integral sliding mode surfaces
$S_{i},(i=4,5,6)$ are selected in each step to improve the robustness of the system. The fuzzy system $U_{i f s},(i=2,3,4)$ are utilized to approximate $U_{i},(i=2,3,4)$ with $\alpha_{i},(i=4,5,6)$ and $\xi_{i},(i=4,5,6)$ being adjustable parameters and fuzzy basis vectors, respectively. Then, the switching control law $U_{i v s},(i=2,3,4)$ are introduced to compensate for $U_{i},(i=2,3,4)$ and the ideal input. $E_{i}, \quad(i=$ $4,5,6)$ are the switching gain. $c_{i}, \eta_{i}, \rho_{i},(i=4,5,6)$, and $k_{i},(i=7,8, \ldots, 12)$ are positive constant. 

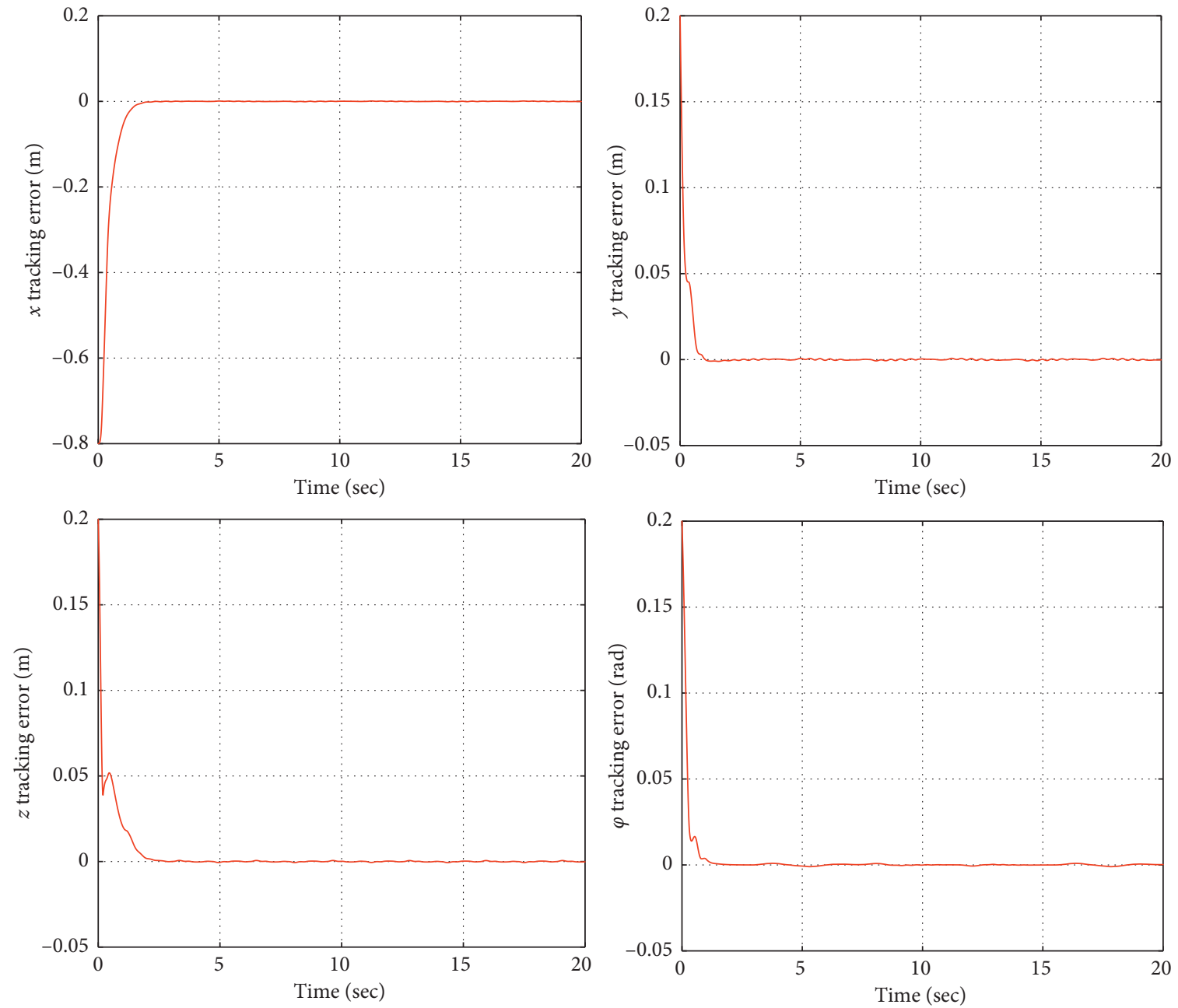

FIGURE 5: The actual tracking error.

Remark 1. $v_{1}, v_{2}$, and $v_{3}$ are combinations of available terms that can be given directly or measured in Tables 1 and 2 . Therefore, the control input $U_{1}$ can be solved by $U_{1}=v_{3} / C_{X_{7}} C_{X_{9}}$.

\section{Stability Analysis of the Closed-Loop System}

In this section, stability analysis of the proposed control system is established to confirm that all signals in the closed loop are ultimately bounded. The errors of the first-order filter are presented as follows:

$$
y_{i}=x_{\mathrm{id}}-\bar{x}_{i}, \quad(i=2,4, \ldots, 12) .
$$

From (T1.3), (T1.9), (T1.15), (T2.3), (T2.9), and (T2.15), one can obtain

$$
\dot{x}_{\mathrm{id}}=-\frac{y_{i}}{\tau_{i / 2}}, \quad(i=2,4, \ldots, 12) .
$$

The derivative of $y_{i}(i=2)$ in time can be obtained as

$$
\dot{y}_{2}=\dot{x}_{2 d}-\bar{x}_{2}=-\frac{y_{2}}{\tau_{1}}-\ddot{x}_{2 d}+c_{1} \dot{e}_{1},
$$

then, one can obtain

$$
\dot{y}_{2}=-\frac{y_{2}}{\tau_{1}}+B_{2}\left(e_{1}, e_{2}, y_{2}, \ddot{x}_{2 d}\right),
$$

where $B_{2}\left(e_{1}, e_{2}, y_{2}, \ddot{x}_{2 d}\right)=-\ddot{x}_{2 d}+c_{1} \dot{e}_{1}$ is a continuous function. The following formula can also be obtained:

$$
\dot{y}_{i}=-\frac{y_{i}}{\tau_{i / 2}}+B_{i}(\cdot), \quad(i=2,4,6,8,10,12) .
$$

Then, the following inequalities hold:

$$
y_{i} \dot{y}_{i} \leq-\frac{y_{i}^{2}}{\tau_{i / 2}}+B_{i}\left|y_{i}\right|, \quad(i=2,4,6,8,10,12) .
$$

Consider the Lyapunov function candidate

$$
V=V_{1}+V_{2} \text {, }
$$

with

$$
V_{1}=(1 / 2) \sum_{i=1}^{6}\left(e_{2 i-1}^{2}+y_{2 i}^{2}\right)
$$
$V_{2}=(1 / 2) \sum_{i=1}^{6}\left(S_{i}^{2}+\left(1 / \eta_{i}\right) \widetilde{\alpha}_{i}^{T} \widetilde{\alpha}_{i}+\left(1 / \rho_{i}\right) \widetilde{E}_{i}^{2}\right)$. Then, the following theorem can be obtained. 

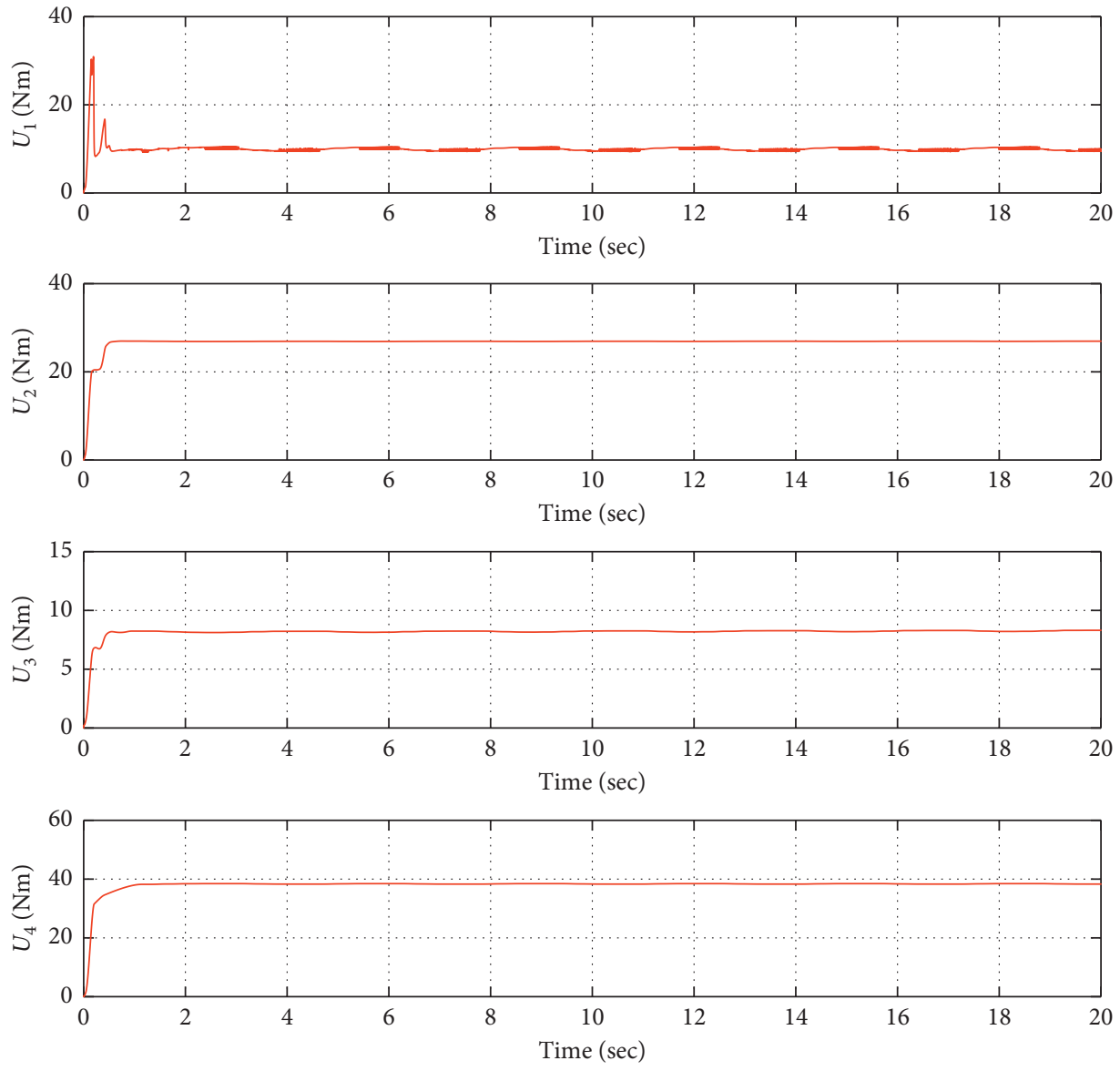

Figure 6: Control inputs.
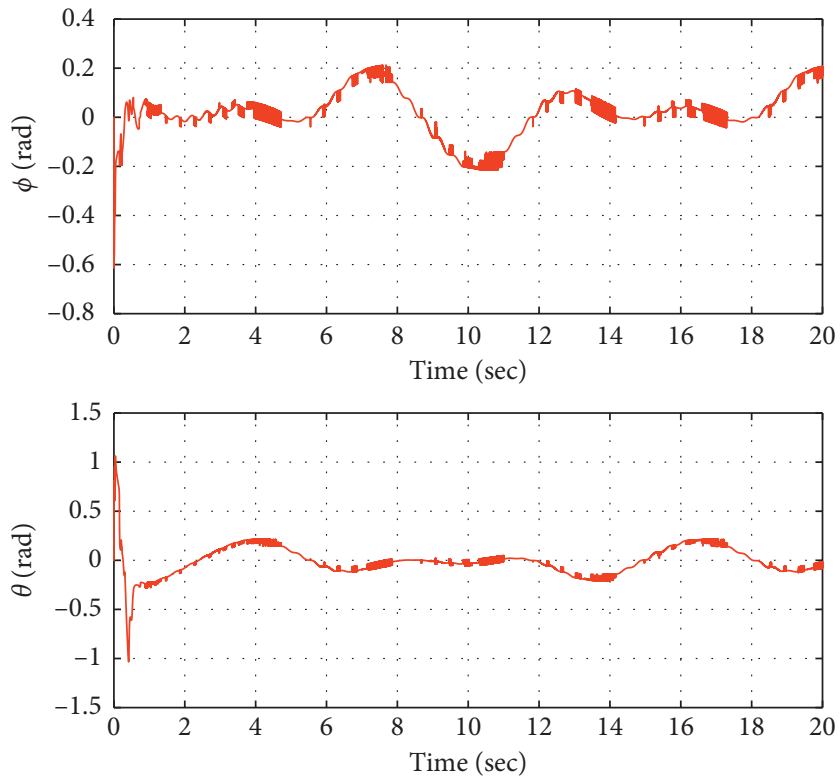

Figure 7: Change of roll and pitch. 


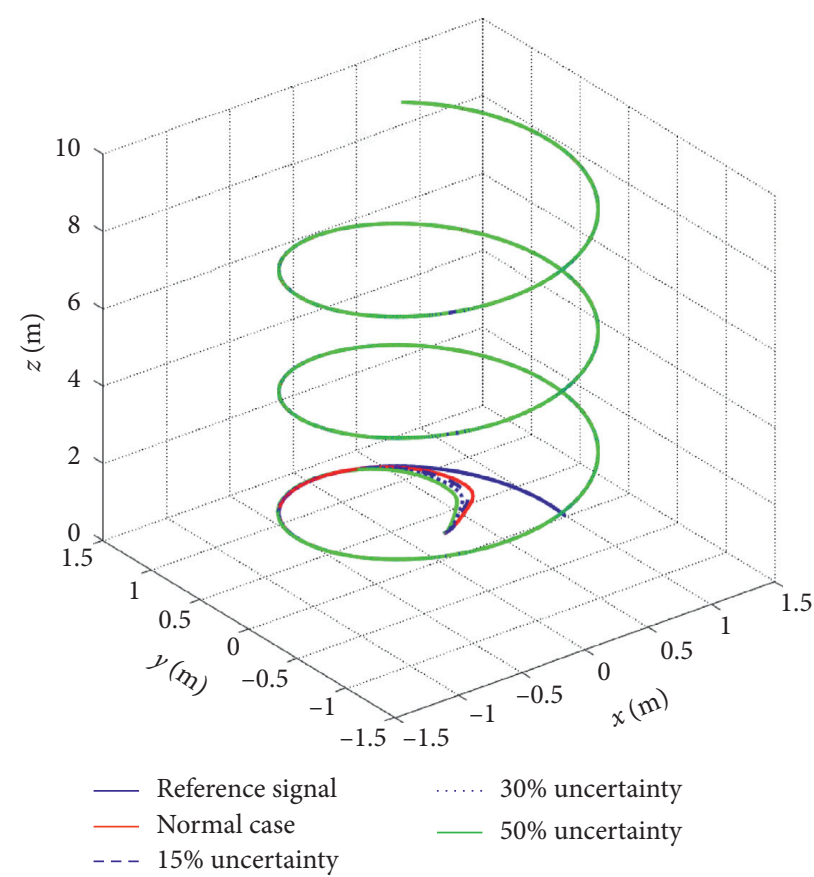

FIgURE 8: Space diagram of position of different uncertainty cases added in yaw axis.
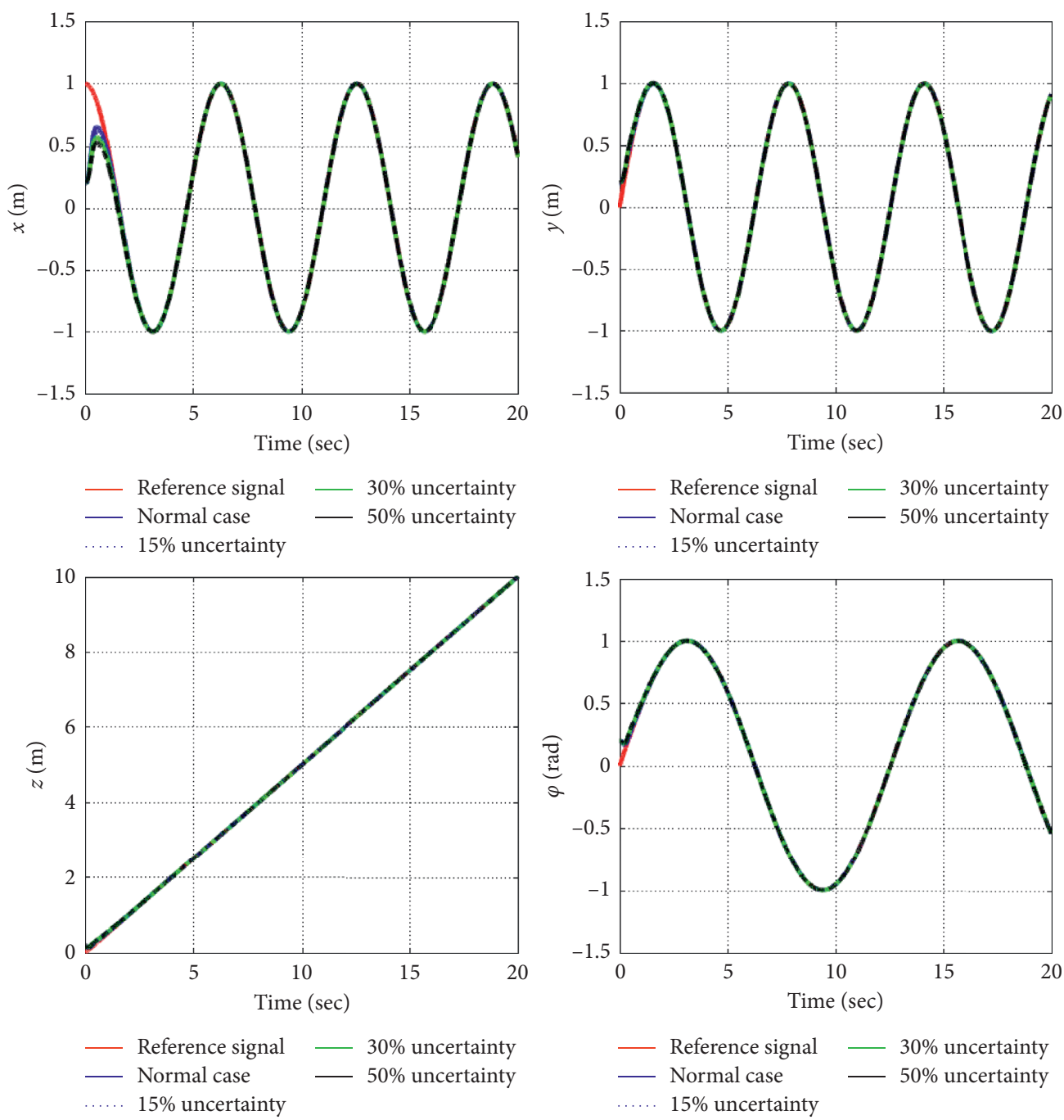

FIGURE 9: Reference singnal and the actual outputs of different uncertainty cases added in yaw axis. 

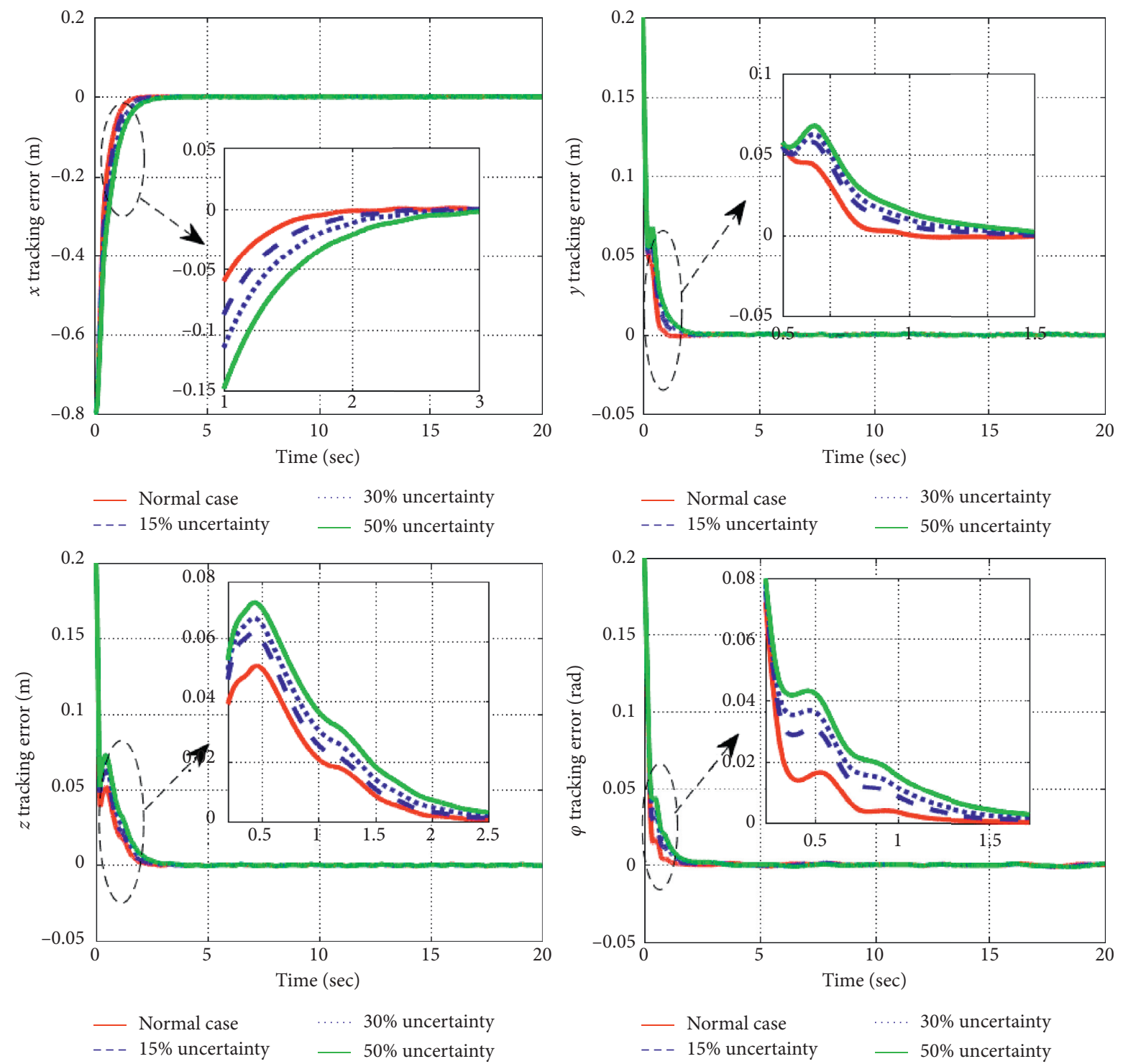

Figure 10: The actual tracking error of different uncertainty cases addes in yaw axis.

Theorem 1. Consider the closed-loop system which consists of the position and attitude system, with the virtual control signals (T1.2), (T1.8), (T1.14), (T2.2), (T2.8), and (T2.14), the adaptive laws (T1.5), (T1.11), (T1.17), (T2.5), (T2.11), and (T2.17), and the switching control laws (T1.6), (T1.12), (T1.18), (T2.6), (T2.12), and (T2.18). If all the parametersc $c_{i}$, $\eta_{i}, \rho_{i},(i=1,2, \ldots, 6), k_{i},(i=1,2, \ldots, 12)$ and the time constant of first-order filter $\tau_{i},(i=1,2, \ldots, 6)$ are designed properly to satisfy $V(0) \leq p,(p>0)$, all the closed-loop signals are uniformly bounded and the tracking error can be kept arbitrarily small.

Proof. The specific proof process is presented in Appendix B.

\section{Simulations}

In this section, the following simulations are given to validate the effectiveness and the performance of the proposed adaptive dynamic surface integral sliding mode control. The parameters for the quadrotor UAV adopted in this paper are presented in Table 3. In the following simulation, the desired trajectory of the position and yaw angle $\{x(t), y(t), z(t), \varphi(t)\}$ are chosen as $\{\sin (t), \cos (t), 0.5 t, \sin (0.5 t)\}$. The controller parameters chosen for simulation are $c_{i}=0.01, \tau_{i}=0.001, \eta_{i}=200$, $\rho_{i}=0.1,(i=1,2, \ldots, 6), \quad k_{i}=9,(i=1,3,5,7,9,11)$, and $k_{i}=20,(i=2,4,6,8,10,12)$. The fuzzy membership functions are chosen as follows: $u_{l}\left(s_{i}\right)=\exp \left(-\left[\left(s_{i}+(\pi / 6)-(l-1) \times(\pi / 12)\right) /\right.\right.$ $\left.(\pi / 24)]^{2}\right),(i=1,2,3 ; l=1,2,3,4,5,6)$. The disturbances are chosen as follows: $d_{1}=\cos (t), \quad d_{2}=\sin (t)$, $d_{3}=\sin (t) \cos (t), \quad d_{4}=0.5 \sin (0.5 t), \quad d_{5}=0.5 \cos (0.5 t)$, $d_{6}=0.25 \sin (0.5 t) \cos (0.5 t)$.

Case 1. In this case, the parameters of the quadrotor are assumed normal. The simulations for this case are presented. 


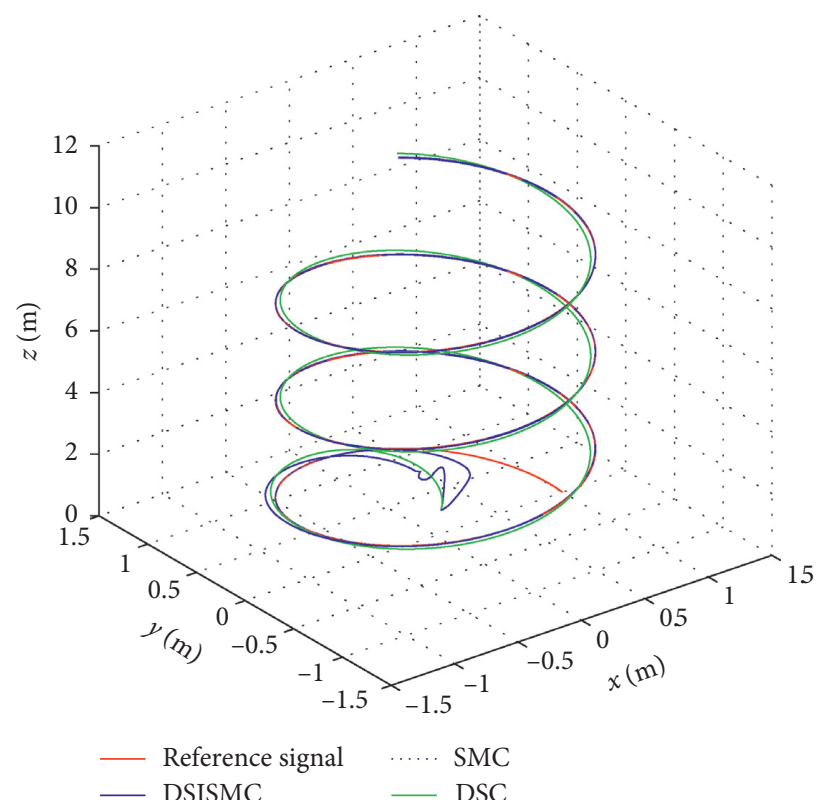

FIGURE 11: Space diagram of position by using DSISMC, SMC, and DSC.
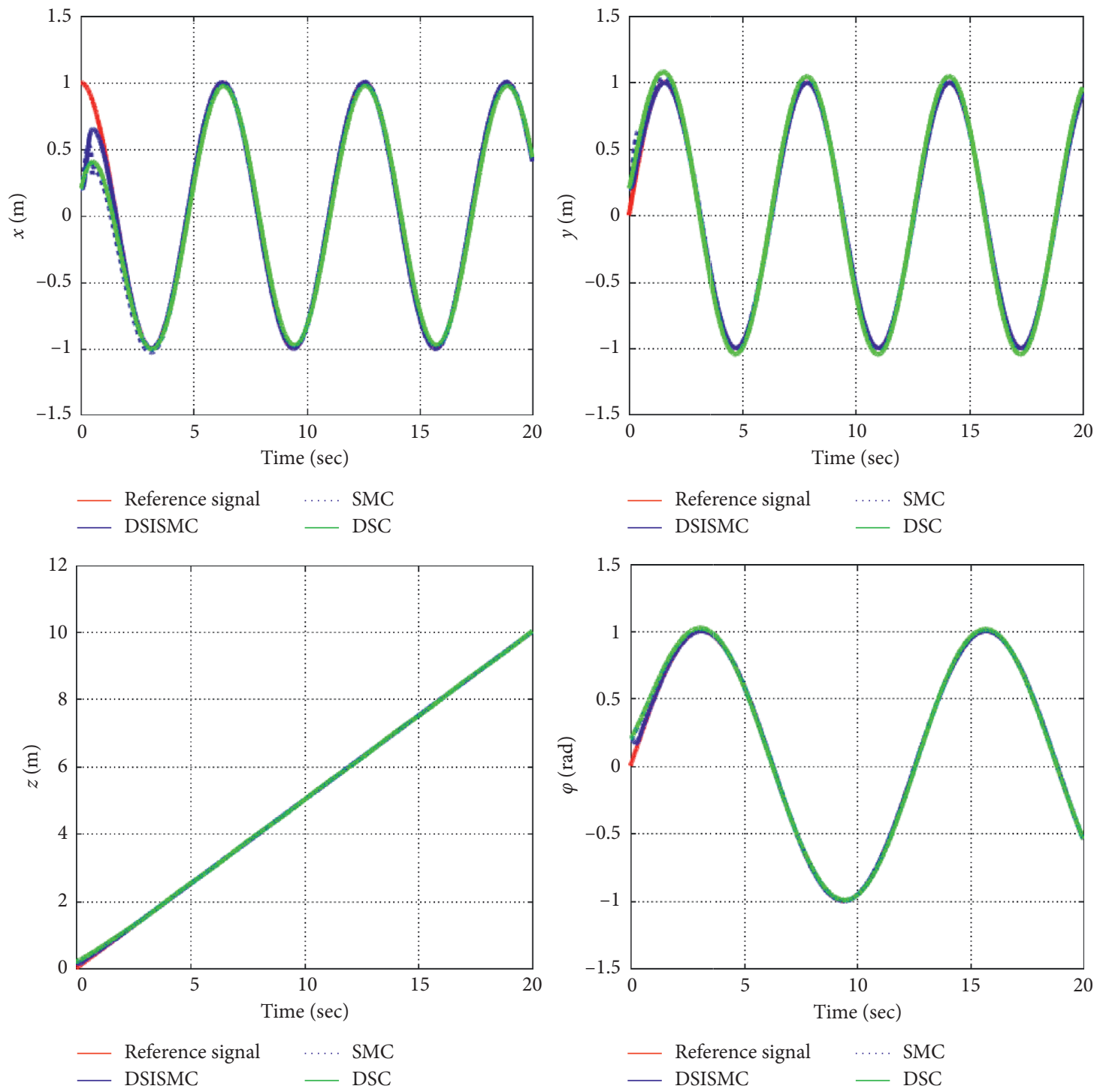

SMC

DSISMC

DSC

FIgURE 12: Reference signal and the actual outputs by using DSISMC, SMC and DSC. 

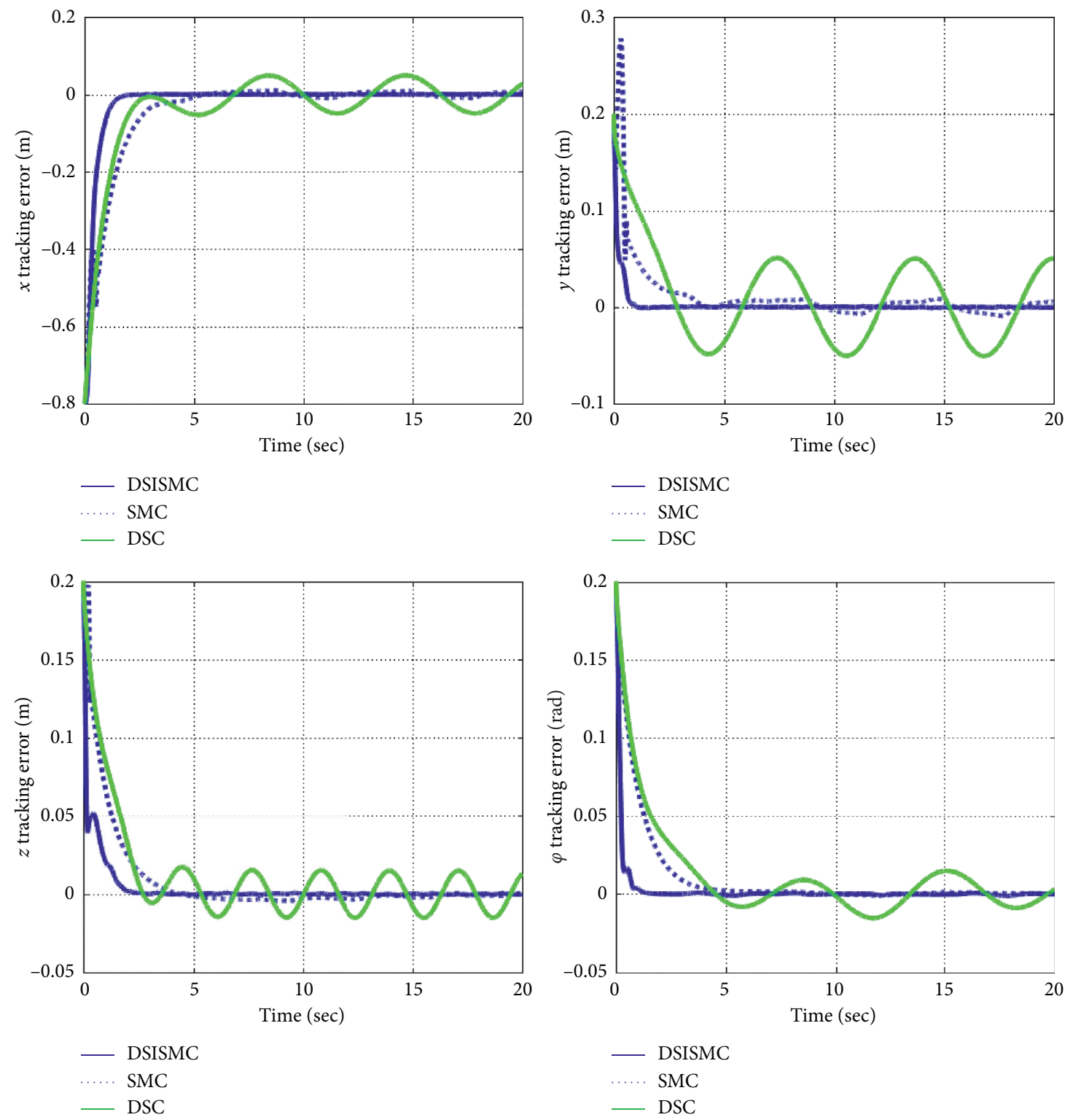

Figure 13: The actual tracking error by using DSISMC, SMC, DSC.

TABle 4: The MVTE and RMSVTE of different schemes.

\begin{tabular}{|c|c|c|c|}
\hline Kind of errors & Proposed scheme & ADSC scheme & ASMC scheme \\
\hline MVTE of $x(\mathrm{~m})$ & $7.66 e^{-4}$ & $4.90 e^{-2}$ & $9.76 e^{-3}$ \\
\hline MVTE of $y(\mathrm{~m})$ & $6.58 e^{-4}$ & $5.03 e^{-2}$ & $7.15 e^{-3}$ \\
\hline MVTE of $z(\mathrm{~m})$ & $5.70 e^{-4}$ & $1.50 e^{-2}$ & $3.15 e^{-3}$ \\
\hline MVTE of $\varphi$ (rad) & $4.83 e^{-4}$ & $1.53 e^{-2}$ & $7.84 e^{-4}$ \\
\hline RMSVTE of $x(\mathrm{~m})$ & $3.33 e^{-4}$ & $3.81 e^{-2}$ & $7.02 e^{-3}$ \\
\hline RMSVTE of $y(\mathrm{~m})$ & $2.92 e^{-4}$ & $4.03 e^{-2}$ & $5.16 e^{-3}$ \\
\hline RMSVTE of $z(\mathrm{~m})$ & $2.16 e^{-4}$ & $9.82 e^{-3}$ & $2.73 e^{-3}$ \\
\hline RMSVTE of $\varphi(\mathrm{rad})$ & $1.34 e^{-4}$ & $1.16 e^{-2}$ & $5.04 e^{-4}$ \\
\hline
\end{tabular}


Cases $2-4$. Uncertainty (15\%, 30\%, and 50\% added) in the rotary inertia as well as the presence of external disturbance. In these cases, we give a consideration to both external disturbance and uncertainty in rotary inertia. The uncertainty in yaw axis is $15 \%, 30 \%$, and $50 \%$, respectively.

The simulation results of Case 1 are presented from Figures 3 to 7 . Figure 3 shows the 3D tracking trajectory by using the proposed controller. The position and yaw angle trajectories are shown in Figure 4, and the tracking errors are given in Figure 5. Figure 6 shows the control signals. Figure 7 shows the change of roll and pitch angles. From the tracking performance, it can be concluded that the proposed control scheme can guarantee that all the variables are bounded and that the control system has strong robustness. Figures 8 to 10 illustrate the tracking performance under the different uncertainty cases. The proposed scheme has the better robustness against external disturbances and uncertainty parameters. Figures 11 to 13 show the tracking error comparisons between the ADSISMC, the ADSC, and the ASMC methods. Meanwhile, the maximum values (MVTE) and the root mean square values (RMSVTE) of tracking error in steady of the proposed scheme and the other two schemes are given in Table 4 . The simulation results show that the proposed scheme has better tracking performance and robustness compared with the ADSC and the ASMC methods.

\section{Conclusion}

This paper proposed a dynamic surface integral sliding mode control scheme for a quadrotor UAV under the conditions of parameter uncertainty and external disturbances. Virtual control inputs are introduced in the robust controller design to guarantee the trajectory tracking performance, and the problem of "explosion of complexity" in the backstepping design has been greatly simplified. The fuzzy systems are utilized to approximate the ideal control inputs and the switch control is introduced to compensate for errors between estimated and ideal inputs which improves the control performance and robustness of the whole system. In addition, the stability analysis of the overall system through Lyapunov stability theory is presented, and all signals of the closed loop are ultimately bounded. Finally, the simulation results show that robustness and improved tracking performance can be achieved with the proposed control scheme.

\section{Appendix}

\section{A. The Controller Design Procedures}

Step 1. Define the position error:

$$
e_{1}=x_{1}-x_{1 d}
$$

where $x_{1 d}$ is the desired $x$ position command, and the derivative of $e_{1}$ with respect to time is

$$
\dot{e}_{1}=x_{2}-\dot{x}_{1 d}
$$

Define the virtual control $\bar{x}_{2}$,

$$
\bar{x}_{2}=-c_{1} e_{1}+\dot{x}_{1 d},
$$

where $c_{1}$ is a positive constant. To solve the problem of "complexity of explosion" caused by the repeated differentiation, a new state variable $x_{2 d}$ is introduced and let $\bar{x}_{2}$ pass through the following first-order filter with constant $\tau_{1}$ (T1.3) to obtain $x_{2 d}$

$$
\tau_{1} \dot{x}_{2 d}+x_{2 d}=\bar{x}_{2}, x_{2 d}(0)=\bar{x}_{2}(0) \text {, }
$$

where $x_{2 d}$ is the output of the first-order filter; the filter error is $y_{1}=x_{2 d}-\bar{x}_{2}$. A proper integral sliding mode manifold is chosen (T1.4):

$$
S_{1}=x_{2}-\int_{0}^{t}\left(\dot{x}_{2 d}-k_{1} \dot{e}_{1}-k_{2} e_{1}\right) \mathrm{d} t
$$

where $k_{1}$ and $k_{2}$ are both the positive constant. If the sliding mode control is in an ideal state, the derivative of $S_{1}$ with respect to time is

$$
S_{1}=\dot{S}_{1}=\dot{x}_{2}-\dot{x}_{2 d}+k_{1} \dot{e}_{1}+k_{2} e_{1}=\dot{e}_{2}+k_{1} \dot{e}_{1}+k_{2} e_{1}=0 .
$$

Then, a variable $v_{1}=\left(C_{x 7} S_{x 9} C_{x 11}+S_{x 7} S_{x 11}\right) U_{1}$ is introduced to be a new control input; then

$$
v_{1}-a_{1} x_{2}+d_{1}-\dot{x}_{2 d}+k_{1} \dot{e}_{1}+k_{2} e_{1}=\dot{e}_{2}+k_{1} \dot{e}_{1}+k_{2} e_{1}=0 .
$$

Assuming that the perturbations and parameters in the equation are known, the control law for $x$ motion in an ideal state is designed as follows:

$$
v_{1}^{*}=a_{1} x_{2}-d_{1}+\dot{x}_{2 d}-k_{1} \dot{e}_{1}-k_{2} e_{1} .
$$

In most cases, the system parameters are uncertain and there are also unknown external disturbances, which makes it difficult to obtain the ideal control signal $v_{1}^{*}$. Therefore, the fuzzy system is used to approximate the ideal control signal $v_{1}^{*}$ and obtain

$$
v_{1}^{*}=v_{1 f z}\left(s_{1}, \alpha_{1}\right)+\varepsilon_{1}=\alpha_{1}^{T} \xi_{1}+\varepsilon_{1},
$$

where $\varepsilon_{1}$ is the approximation error, and $\left|\varepsilon_{1}\right|<E_{1}$. Introducing switching control law $v_{1 v s}$ (T1.5) to compensate for $v_{1}^{*}$ and $v_{1 f z}$,

$$
v_{1 v s}=-\widehat{E}_{1} \operatorname{sgn}\left(S_{1}\right)
$$

where $\widehat{E}_{1}$ is the estimation of $E_{1}$.

Then, the actual control law (T1.6) is obtained as

$$
v_{1}=v_{1 f z}+v_{1 v s}
$$

Consider the Lyapunov function

$$
\Gamma_{1}=\frac{1}{2} S_{1}^{2}+\frac{1}{2 \eta_{1}} \widetilde{\alpha}_{1}^{T} \widetilde{\alpha}_{1}+\frac{1}{2 \rho_{1}} \widetilde{E}_{1}^{2},
$$

where $\eta_{1}$ and $\rho_{1}$ are positive constant. The derivative of $\Gamma_{1}$ with respect to time can be presented below: 


$$
\dot{\Gamma}_{1}=S_{1} \dot{S}_{1}+\frac{1}{\eta_{1}} \widetilde{\alpha}_{1}^{T} \dot{\tilde{\alpha}}_{1}+\frac{1}{\rho_{1}} \widetilde{E}_{1} \dot{\tilde{E}}_{1} \text {, }
$$

while

$$
\begin{aligned}
v_{1}^{*} & =a_{1} x_{2}-d_{1}+\dot{x}_{2 d}-k_{1} \dot{e}_{1}-k_{2} e_{1} \\
& =a_{1} x_{2}-d_{1}+\dot{x}_{2}-\dot{S}_{1} \\
& =v_{1}-\dot{S}_{1} .
\end{aligned}
$$

So

$$
\dot{S}_{1}=v_{1}-v_{1}^{*}=v_{1 f z}+v_{1 v s}-v_{1}^{*}
$$

Substituting (A.15) into (A.13), then

$$
\begin{aligned}
\dot{\Gamma}_{1} & =S_{1} \dot{S}_{1}+\frac{1}{\eta_{1}} \widetilde{\alpha}_{1}^{T} \dot{\tilde{\alpha}}_{1}+\frac{1}{\rho_{1}} \widetilde{E}_{1} \dot{\widetilde{E}}_{1} \\
& =S_{1}\left(v_{1 f z}+v_{1 v s}-v_{1}^{*}\right)+\frac{1}{\eta_{1}} \widetilde{\alpha}_{1}^{T} \dot{\tilde{\alpha}}_{1}+\frac{1}{\rho_{1}} \widetilde{E}_{1} \dot{\widetilde{E}}_{1} \\
& =\widetilde{\alpha}_{1}^{T}\left(S_{1} \xi_{1}+\frac{1}{\eta_{1}} \dot{\tilde{\alpha}}_{1}\right)+S_{1}\left(v_{1 v s}-\varepsilon_{1}\right)+\frac{1}{\rho_{1}} \widetilde{E}_{1} \dot{\widetilde{E}}_{1} .
\end{aligned}
$$

The adaptive law and the switching control (T1.5) are chosen below:

$$
\dot{\widehat{\alpha}}_{1}=-\eta_{1} S_{1} \xi_{1} \text {. }
$$

And formula (A.16) becomes

$$
\begin{aligned}
\dot{\Gamma}_{1} & =S_{1} \dot{S}_{1}+\frac{1}{\eta_{1}} \widetilde{\alpha}_{1}^{T} \dot{\tilde{\alpha}}_{1}+\frac{1}{\rho_{1}} \widetilde{E}_{1} \dot{\widetilde{E}}_{1} \\
& =\widetilde{\alpha}_{1}^{T}\left(S_{1} \xi_{1}+\frac{1}{\eta_{1}} \dot{\tilde{\alpha}}_{1}\right)+S_{1}\left(v_{1 v s}-\varepsilon_{1}\right)+\frac{1}{\rho_{1}} \widetilde{E}_{1} \dot{\widetilde{E}}_{1} \\
& =-\widehat{E}_{1}\left|S_{1}\right|-\varepsilon_{1} S_{1}+\frac{1}{\rho_{1}}\left(\widehat{E}_{1}-E_{1}\right) \dot{\widehat{E}}_{1} .
\end{aligned}
$$

To make the Lyapunov function $\dot{\Gamma}_{1} \leq 0$, the adaptive law of switching control is updated below:

$$
\dot{\widehat{E}}_{1}=\rho_{1}\left|S_{1}\right| \text {. }
$$

Then,

$$
\begin{aligned}
\dot{\Gamma}_{1} & =-\widehat{E}_{1}\left|S_{1}\right|-\varepsilon_{1} S_{1}+\frac{1}{\rho_{1}}\left(\widehat{E}_{1}-E_{1}\right) \dot{\widehat{E}}_{1} \\
& \leq-E_{1}\left|S_{1}\right|-\varepsilon_{1} S_{1} \\
& \leq-\left(E_{1}-\left|\varepsilon_{1}\right|\right)\left|S_{1}\right| \\
& \leq 0
\end{aligned}
$$

The similar design processes are presented to design the trajectory tracking of $y$-axis position $x_{3}$ and $z$-axis position $x_{5}$ using the dynamic surface integral sliding mode control. Introduce the variables $v_{2}=\left(C_{x 7} S_{x 9} S_{x 11}-S_{x 7} S_{x 11}\right) U_{1}$ and $v_{3}=\left(C_{x 7} C_{x 9}\right) U_{1}$, the specific procedures are presented in Step 2 and Step 3.

Step 2. Define the position error:

$$
e_{3}=x_{3}-x_{3 d}
$$

where $x_{3 d}$ is the desired $y$-position command, and the derivative of $e_{3}$ with respect to time is

$$
\dot{e}_{3}=x_{4}-\dot{x}_{3 d} \text {. }
$$

Define the virtual control $\bar{x}_{4}$,

$$
\bar{x}_{4}=-c_{2} e_{3}+\dot{x}_{3 d}
$$

where $c_{2}$ is a positive constant. A new state variable $x_{4 d}$ is introduced and let $\bar{x}_{4}$ pass through the following first-order filter with constant $\tau_{2}$ (T1.9) to obtain $x_{4 d}$

$$
\tau_{2} \dot{x}_{4 d}+x_{4 d}=\bar{x}_{4}, x_{4 d}(0)=\bar{x}_{4}(0)
$$

where $x_{4 d}$ is the output of the first-order filter; the filter error is $y_{4}=x_{4 d}-\bar{x}_{4}$. A proper integral sliding mode manifold is chosen (T1.10):

$$
S_{2}=x_{4}-\int_{0}^{t}\left(\dot{x}_{4 d}-k_{3} \dot{e}_{3}-k_{4} e_{3}\right) \mathrm{d} t
$$

where $k_{3}$ and $k_{4}$ are the positive constant. If the sliding mode control is in an ideal state, and the derivative of $S_{2}$ with respect to time is

$$
S_{2}=\dot{S}_{2}=\dot{x}_{4}-\dot{x}_{4 d}+k_{3} \dot{e}_{3}+k_{4} e_{3}=\dot{e}_{4}+k_{3} \dot{e}_{3}+k_{4} e_{3}=0 .
$$

Then, a variable $v_{2}=\left(C_{x 7} S_{x 9} S_{x 11}-S_{x 7} S_{x 11}\right) U_{1}$ is introduced to be a new control input. Assuming that the perturbations and parameters in the equation are known, the control law for $y$-motion in an ideal state is designed as follows:

$$
v_{2}^{*}=a_{2} x_{4}-d_{2}+\dot{x}_{4 d}-k_{3} \dot{e}_{3}-k_{4} e_{3} .
$$

In most cases, the system parameters are uncertain and there are also unknown external disturbances, which makes it difficult to obtain the ideal control $\operatorname{signal} v_{2}^{*}$. Therefore, the fuzzy system is used to approximate the ideal control signal $v_{2}^{*}$ and obtain

$$
v_{2}^{*}=v_{2 f z}\left(s_{2}, \alpha_{2}\right)+\varepsilon_{2}=\alpha_{2}^{T} \xi_{2}+\varepsilon_{2},
$$

where $\varepsilon_{2}$ is the approximation error, and $\left|\varepsilon_{2}\right|<E_{2}$. Introducing switching control law $v_{2 v s}$ to compensate for $v_{2}^{*}$ and $v_{2 f z}$ 


$$
v_{2 v s}=-\widehat{E}_{2} \operatorname{sgn}\left(S_{2}\right),
$$

where $\widehat{E}_{2}$ is the estimation of $E_{2}$. Then, the actual control law (T1.12) is obtained

$$
v_{2}=v_{2 f z}+v_{2 v s} .
$$

Consider the Lyapunov function

$$
\Gamma_{2}=\frac{1}{2} S_{2}^{2}+\frac{1}{2 \eta_{2}} \widetilde{\alpha}_{2}^{T} \widetilde{\alpha}_{2}+\frac{1}{2 \rho_{2}} \widetilde{E}_{2}^{2},
$$

where $\eta_{2}$ and $\rho_{2}$ are positive constant. The derivative of $\Gamma_{2}$ with respect to time can be presented as below:

$$
\dot{\Gamma}_{2}=S_{2} \dot{S}_{2}+\frac{1}{\eta_{2}} \widetilde{\alpha}_{2}^{T} \dot{\tilde{\alpha}}_{2}+\frac{1}{\rho_{2}} \widetilde{E}_{2} \dot{\tilde{E}}_{2},
$$

while

$$
\begin{aligned}
v_{2}^{*} & =a_{2} x_{4}-d_{2}+\dot{x}_{4 d}-k_{3} \dot{e}_{3}-k_{4} e_{3} \\
& =a_{2} x_{4}-d_{2}+\dot{x}_{4}-\dot{S}_{2} \\
& =v_{2}-\dot{S}_{2} .
\end{aligned}
$$

So,

$$
\dot{S}_{2}=v_{2}-v_{2}^{*}=v_{2 f z}+v_{2 v s}-v_{2}^{*} .
$$

Substituting (A.34) into (A.32), then

$$
\begin{aligned}
\dot{\Gamma}_{2} & =S_{2} \dot{S}_{2}+\frac{1}{\eta_{2}} \widetilde{\alpha}_{2}^{T} \dot{\tilde{\alpha}}_{2}+\frac{1}{\rho_{2}} \widetilde{E}_{2} \dot{\tilde{E}}_{2} \\
& =S_{2}\left(v_{2 f z}+v_{2 v s}-v_{2}^{*}\right)+\frac{1}{\eta_{2}} \widetilde{\alpha}_{2}^{T} \dot{\tilde{\alpha}}_{2}+\frac{1}{\rho_{2}} \widetilde{E}_{2} \dot{\tilde{E}}_{2} \\
& =\widetilde{\alpha}_{2}^{T}\left(S_{2} \xi_{2}+\frac{1}{\eta_{2}} \dot{\widetilde{\alpha}}_{2}\right)+S_{2}\left(v_{2 v s}-\varepsilon_{2}\right)+\frac{1}{\rho_{2}} \widetilde{E}_{2} \dot{\tilde{E}}_{2} .
\end{aligned}
$$

The adaptive law (T1.11) is chosen below:

$$
\dot{\widehat{\alpha}}_{2}=-\eta_{2} S_{2} \xi_{2} \text {. }
$$

And formula (A.35) becomes

$$
\begin{aligned}
\dot{\Gamma}_{2} & =S_{2} \dot{S}_{2}+\frac{1}{\eta_{2}} \widetilde{\alpha}_{2}^{T} \dot{\tilde{\alpha}}_{2}+\frac{1}{\rho_{2}} \widetilde{E}_{2} \dot{\tilde{E}}_{2} \\
& =\widetilde{\alpha}_{2}^{T}\left(S_{2} \xi_{2}+\frac{1}{\eta_{2}} \dot{\widetilde{\alpha}}_{2}\right)+S_{2}\left(v_{2 v s}-\varepsilon_{2}\right)+\frac{1}{\rho_{2}} \widetilde{E}_{2} \dot{\widetilde{E}}_{2} \\
& =-\widehat{E}_{2}\left|S_{2}\right|-\varepsilon_{2} S_{2}+\frac{1}{\rho_{2}}\left(\widehat{E}_{2}-E_{2}\right) \dot{\hat{E}}_{2} .
\end{aligned}
$$

To make the Lyapunov function $\dot{\Gamma}_{2} \leq 0$, the adaptive law of switching control is updated below:

$$
\dot{\widehat{E}}_{2}=\rho_{2}\left|S_{2}\right| \text {. }
$$

Then,

$$
\begin{aligned}
\dot{\Gamma}_{2} & =-\widehat{E}_{2}\left|S_{2}\right|-\varepsilon_{2} S_{2}+\frac{1}{\rho_{2}}\left(\widehat{E}_{2}-E_{2}\right) \dot{\hat{E}}_{2} \\
& \leq-E_{2}\left|S_{2}\right|-\varepsilon_{2} S_{2} \\
& \leq-\left(E_{2}-\left|\varepsilon_{2}\right|\right)\left|S_{2}\right| \\
& \leq 0 .
\end{aligned}
$$

Step 3. Define the position error:

$$
e_{5}=x_{5}-x_{5 d}
$$

where $x_{5 d}$ is the desired $z$-position command, and the derivative of $e_{5}$ with respect to time is

$$
\dot{e}_{5}=x_{6}-\dot{x}_{5 d} .
$$

Define the virtual control $\bar{x}_{6}$,

$$
\bar{x}_{6}=-c_{3} e_{5}+\dot{x}_{5 d},
$$

where $c_{3}$ is a positive constant. A new state variable $x_{6 d}$ is introduced and let $\bar{x}_{6}$ pass through the following first-order filter with constant $\tau_{3}$ (T1.15) to obtain $x_{6 d}$

$$
\begin{aligned}
\tau_{3} \dot{x}_{6 d}+x_{6 d} & =\bar{x}_{6}, \\
x_{6 d}(0) & =\bar{x}_{6}(0),
\end{aligned}
$$

where $x_{6 d}$ is the output of the first-order filter; the filter error is $y_{6}=x_{6 d}-\bar{x}_{6}$. A proper integral sliding mode manifold is chosen (T1.16):

$$
S_{3}=x_{6}-\int_{0}^{t}\left(\dot{x}_{6 d}-k_{5} \dot{e}_{5}-k_{6} e_{5}\right) \mathrm{d} t
$$

where $k_{5}$ and $k_{6}$ are the positive constant. If the sliding mode control is in an ideal state, and the derivative of $S_{3}$ with respect to time is

$$
S_{3}=\dot{S}_{3}=\dot{x}_{6}-\dot{x}_{6 d}+k_{5} \dot{e}_{5}+k_{6} e_{5}=\dot{e}_{6}+k_{5} \dot{e}_{5}+k_{6} e_{5}=0 .
$$

Then, a variable $v_{3}=\left(C_{x 7} C_{x 9}\right) U_{1}$ is introduced to be a new control input. Assuming that the perturbations and parameters in the equation are known, the control law for $z$ motion in an ideal state is designed as follows:

$$
v_{3}^{*}=a_{3} x_{6}-d_{3}+g+\dot{x}_{6 d}-k_{5} \dot{e}_{5}-k_{6} e_{5} .
$$

In most cases, the system parameters are uncertain and there are also unknown external disturbances, which makes it difficult to obtain the ideal control $\operatorname{signal} v_{3}^{*}$. Therefore, the fuzzy system is used to approximate the ideal control signal $v_{3}^{*}$ and obtain

$$
v_{3}^{*}=v_{3 f z}\left(s_{3}, \alpha_{3}\right)+\varepsilon_{3}=\alpha_{3}^{T} \xi_{3}+\varepsilon_{3},
$$

where $\varepsilon_{3}$ is the approximation error, and $\left|\varepsilon_{3}\right|<E_{3}$. Introducing switching control law $v_{3 v s}$ (T1.17) to compensate for $v_{3}^{*}$ and $v_{3 f z}$ 


$$
v_{3 v s}=-\widehat{E}_{3} \operatorname{sgn}\left(S_{3}\right),
$$

where $\widehat{E}_{3}$ is the estimation of $E_{3}$. Then, the actual control law (T1.18) is obtained as

$$
v_{3}=v_{3 f z}+v_{3 v s}
$$

Consider the Lyapunov function

$$
\Gamma_{3}=\frac{1}{2} S_{3}^{2}+\frac{1}{2 \eta_{3}} \widetilde{\alpha}_{3}^{T} \widetilde{\alpha}_{3}+\frac{1}{2 \rho_{3}} \widetilde{E}_{3}^{2},
$$

where $\eta_{3}$ and $\rho_{3}$ are the positive constant. The derivative of $\Gamma_{3}$ with respect to time can be presented as below:

$$
\dot{\Gamma}_{3}=S_{3} \dot{S}_{3}+\frac{1}{\eta_{3}} \widetilde{\alpha}_{3}^{T} \dot{\tilde{\alpha}}_{3}+\frac{1}{\rho_{3}} \widetilde{E}_{3} \dot{\tilde{E}}_{3},
$$

while

$$
\begin{aligned}
v_{3}^{*} & =a_{3} x_{6}-d_{3}+g+\dot{x}_{6 d}-k_{5} \dot{e}_{5}-k_{6} e_{5} \\
& =a_{3} x_{6}-d_{3}+g+\dot{x}_{6}-\dot{S}_{3} \\
& =v_{3}-\dot{S}_{3} .
\end{aligned}
$$

So,

$$
\dot{S}_{3}=v_{3}-v_{3}^{*}=v_{3 f z}+v_{3 v s}-v_{3}^{*} .
$$

Substituting (A.53) into (A.51), then

$$
\begin{aligned}
\dot{\Gamma}_{3} & =S_{3} \dot{S}_{3}+\frac{1}{\eta_{3}} \widetilde{\alpha}_{3}^{T} \dot{\tilde{\alpha}}_{3}+\frac{1}{\rho_{3}} \widetilde{E}_{3} \dot{\widetilde{E}}_{3} \\
& =S_{3}\left(v_{3 f z}+v_{3 v s}-v_{3}^{*}\right)+\frac{1}{\eta_{3}} \widetilde{\alpha}_{3}^{T} \dot{\tilde{\alpha}}_{3}+\frac{1}{\rho_{3}} \widetilde{E}_{3} \dot{\tilde{E}}_{3} \\
& =\widetilde{\alpha}_{3}^{T}\left(S_{3} \xi_{3}+\frac{1}{\eta_{3}} \dot{\tilde{\alpha}}_{3}\right)+S_{3}\left(v_{3 v s}-\varepsilon_{3}\right)+\frac{1}{\rho_{3}} \widetilde{E}_{3} \dot{\tilde{E}}_{3} .
\end{aligned}
$$

The adaptive law (T1.17) is chosen below:

$$
\dot{\hat{\alpha}}_{3}=-\eta_{3} S_{3} \xi_{3} \text {. }
$$

And formula (A.54) becomes

$$
\begin{aligned}
\dot{\Gamma}_{3} & =S_{3} \dot{S}_{3}+\frac{1}{\eta_{3}} \widetilde{\alpha}_{3}^{T} \dot{\tilde{\alpha}}_{3}+\frac{1}{\rho_{3}} \widetilde{E}_{3} \dot{\tilde{E}}_{3} \\
& =\widetilde{\alpha}_{3}^{T}\left(S_{3} \xi_{3}+\frac{1}{\eta_{3}} \dot{\tilde{\alpha}}_{3}\right)+S_{3}\left(v_{3 v s}-\varepsilon_{3}\right)+\frac{1}{\rho_{3}} \widetilde{E}_{3} \dot{\widetilde{E}}_{3} \\
& =-\widehat{E}_{3}\left|S_{3}\right|-\varepsilon_{3} S_{3}+\frac{1}{\rho_{3}}\left(\widehat{E}_{3}-E_{3}\right) \dot{\widehat{E}}_{3} .
\end{aligned}
$$

To make the Lyapunov function $\dot{\Gamma}_{3} \leq 0$, the adaptive law of switching control is updated below:

$$
\dot{\widehat{E}}_{3}=\rho_{3}\left|S_{3}\right| \text {. }
$$

Then,

$$
\begin{aligned}
\dot{\Gamma}_{3} & =-\widehat{E}_{3}\left|S_{3}\right|-\varepsilon_{3} S_{3}+\frac{1}{\rho_{3}}\left(\widehat{E}_{3}-E_{3}\right) \dot{\hat{E}}_{3} \\
& \leq-E_{3}\left|S_{3}\right|-\varepsilon_{3} S_{3} \\
& \leq-\left(E_{3}-\left|\varepsilon_{3}\right|\right)\left|S_{3}\right| \\
& \leq 0 .
\end{aligned}
$$

By associating $v_{1}, v_{2}$, and $v_{3}$, a group of virtual controls is obtained as follows:

$$
\left\{\begin{array}{l}
v_{1}=\left(C_{x 7} S_{x 9} C_{x 11}+S_{x 7} S_{x 11}\right) U_{1}, \\
v_{2}=\left(C_{x 7} S_{x 9} S_{x 11}-S_{x 7} S_{x 11}\right) U_{1}, \\
v_{3}=\left(C_{x 7} C_{x 9}\right) U_{1} .
\end{array}\right.
$$

Remark 2. $v_{1}, v_{2}$, and $v_{3}$ are combinations of available terms that can be given directly or measured above. Therefore, the control input $U_{1}$ can be solved by regarding them as known in the controlled system (A.59). Apparently, (A.59) has four unknown variables, namely, $x_{7}, x_{9}, x_{11}$, and $U_{1}$. However, $x_{11 d}$ is usually given as an extra reference signal in advance and the integral SMC controller is designed above to ensure the rapid convergence of $x_{11}$ to $x_{11 d}$. Thus, $x_{11}$ is regarded as known and can be replaced by $x_{11 d}$ in this situation, and the unknown variables are reduced. So, we can obtain the unknown variables as follows:

$$
\left\{\begin{array}{l}
x_{7 d}=\arctan \left(C_{x_{9}} \frac{b v_{1}-a v_{2}}{v_{3}}\right), \\
x_{9 d}=\arctan \left(\frac{a v_{1}+b v_{2}}{v_{3}}\right), \\
U_{1}=\frac{v_{3}}{C_{X_{7}} C_{X_{9}}}
\end{array}\right.
$$

where $x_{7 d}$ and $x_{9 d}$ are desired the roll and pitch angle trajectory, and $U_{1}$ is part of the ultimate control laws, $a=$ $\cos \left(x_{11 d}\right)$ and $b=\sin \left(x_{11 d}\right)$.

In the attitude tracking system, $x_{7 d}, x_{9 d}$, and $x_{11 d}$ are taken as the desired attitude trajectory, and the design procedure of attitude tracking contains three steps.

Step 4. Define the roll error:

$$
e_{7}=x_{7}-x_{7 d},
$$

where $x_{7 d}$ is the desired roll command, and the derivative of $e_{7}$ with respect to time is:

$$
\dot{e}_{7}=x_{8}-\dot{x}_{7 d} .
$$

Define the virtual control $\bar{x}_{8}$,

$$
\bar{x}_{8}=-c_{4} e_{7}+\dot{x}_{7 d},
$$


where $c_{4}$ is a positive constant. A new state variable $x_{8 d}$ is introduced and let $\bar{x}_{8}$ pass through the following first-order filter with constant $\tau_{4}$ (T2.3) to obtain $x_{8 d}$

$$
\begin{aligned}
\tau_{4} \dot{x}_{8 d}+x_{8 d} & =\bar{x}_{8}, \\
x_{8 d}(0) & =\bar{x}_{8}(0),
\end{aligned}
$$

where $x_{8 d}$ is the output of the first-order filter; the filter error is $y_{8}=x_{8 d}-\bar{x}_{8}$. A proper integral sliding mode manifold is chosen (T2.4):

$$
S_{4}=x_{8}-\int_{0}^{t}\left(\dot{x}_{8 d}-k_{7} \dot{e}_{7}-k_{8} e_{7}\right) \mathrm{d} t,
$$

where $k_{7}$ and $k_{8}$ are the positive constant. If the sliding mode control is in an ideal state, the derivative of $S_{4}$ with respect to time is

$$
S_{4}=\dot{S}_{4}=\dot{x}_{8}-\dot{x}_{8 d}+k_{7} \dot{e}_{7}+k_{8} e_{7}=\dot{e}_{8}+k_{7} \dot{e}_{7}+k_{8} e_{7}=0 .
$$

Assuming that the perturbations and parameters in the equation are known, the control law for roll motion in an ideal state is designed as follows:

$$
U_{2}^{*}=-a_{4} x_{10} x_{12}-a_{5} \omega x_{10}+a_{6} x_{8}-d_{4}+\dot{x}_{8 d}-k_{7} \dot{e}_{7}-k_{8} e_{7} .
$$

In most cases, the system parameters are uncertain and there are also unknown external disturbances, which makes it difficult to obtain the ideal control signal $U_{2}^{*}$. Therefore, the fuzzy system is used to approximate the ideal control signal $U_{2}^{*}$ and obtain

$$
U_{2}^{*}=U_{2 f z}\left(s_{2}, \alpha_{4}\right)+\varepsilon_{4}=\alpha_{4}^{T} \xi_{4}+\varepsilon_{4},
$$

where $\varepsilon_{4}$ is the approximation error, and $\left|\varepsilon_{4}\right|<E_{4}$. Introducing switching control law $U_{2 v s}$ (T2.5) to compensate for $U_{2}^{*}$ and $U_{2 f z}$

$$
U_{2 v s}=-\widehat{E}_{4} \operatorname{sgn}\left(S_{4}\right) \text {, }
$$

where $\widehat{E}_{4}$ is the estimation of $E_{4}$. Then, the actual control law (T2.6) is obtained as

$$
U_{2}=U_{2 f z}+U_{2 v s}
$$

Consider the Lyapunov function

$$
\Gamma_{4}=\frac{1}{2} S_{4}^{2}+\frac{1}{2 \eta_{4}} \widetilde{\alpha}_{4}^{T} \widetilde{\alpha}_{4}+\frac{1}{2 \rho_{4}} \widetilde{E}_{4}^{2}
$$

where $\eta_{4}$ and $\rho_{4}$ are the positive constant. The derivative of $\Gamma_{4}$ with respect to time can be presented as below:

$$
\dot{\Gamma}_{4}=S_{4} \dot{S}_{4}+\frac{1}{\eta_{4}} \widetilde{\alpha}_{4}^{T} \dot{\tilde{\alpha}}_{4}+\frac{1}{\rho_{4}} \widetilde{E}_{4} \dot{\widetilde{E}}_{4},
$$

while

$$
\begin{aligned}
U_{2}^{*} & =-a_{4} x_{10} x_{12}-a_{5} \oplus x_{10}+a_{6} x_{8}-d_{4}+\dot{x}_{8 d}-k_{7} \dot{e}_{7}-k_{8} e_{7} \\
& =-a_{4} x_{10} x_{12}-a_{5} \oplus x_{10}+a_{6} x_{8}-d_{4}+\dot{x}_{8}-\dot{S}_{4} \\
& =U_{3}-\dot{S}_{4} .
\end{aligned}
$$

So,

$$
\dot{S}_{4}=U_{2}-U_{2}^{*}=U_{2 f z}+U_{2 v s}-U_{2}^{*} .
$$

Substituting (A.74) into (A.72), then

$$
\begin{aligned}
\dot{\Gamma}_{4} & =S_{4} \dot{S}_{4}+\frac{1}{\eta_{4}} \widetilde{\alpha}_{4}^{T} \dot{\tilde{\alpha}}_{4}+\frac{1}{\rho_{4}} \widetilde{E}_{4} \dot{\widetilde{E}}_{4} \\
& =S_{4}\left(U_{2 f z}+U_{2 v s}-U_{2}^{*}\right)+\frac{1}{\eta_{4}} \widetilde{\alpha}_{4}^{T} \dot{\tilde{\alpha}}_{4}+\frac{1}{\rho_{4}} \widetilde{E}_{4} \dot{\widetilde{E}}_{4} \\
& =\widetilde{\alpha}_{4}^{T}\left(S_{4} \xi_{4}+\frac{1}{\eta_{4}} \dot{\tilde{\alpha}}_{4}\right)+S_{4}\left(U_{2 v s}-\varepsilon_{4}\right)+\frac{1}{\rho_{4}} \widetilde{E}_{4} \dot{\widetilde{E}}_{4} .
\end{aligned}
$$

The adaptive law (T2.5) is chosen below:

$$
\dot{\hat{\alpha}}_{4}=-\eta_{4} S_{4} \xi_{4} \text {. }
$$

And formula (A.75) becomes

$$
\begin{aligned}
\dot{\Gamma}_{4} & =S_{4} \dot{S}_{4}+\frac{1}{\eta_{4}} \widetilde{\alpha}_{4}^{T} \dot{\widetilde{\alpha}}_{4}+\frac{1}{\rho_{4}} \widetilde{E}_{4} \dot{\widetilde{E}}_{4} \\
& =\widetilde{\alpha}_{4}^{T}\left(S_{4} \xi_{4}+\frac{1}{\eta_{4}} \dot{\widetilde{\alpha}}_{4}\right)+S_{4}\left(U_{2 v s}-\varepsilon_{4}\right)+\frac{1}{\rho_{4}} \widetilde{E}_{4} \dot{\widetilde{E}}_{4} \\
& =-\widehat{E}_{4}\left|S_{4}\right|-\varepsilon_{4} S_{4}+\frac{1}{\rho_{4}}\left(\widehat{E}_{4}-E_{4}\right) \dot{\widehat{E}}_{4} .
\end{aligned}
$$

To make the Lyapunov function $\dot{\Gamma}_{4} \leq 0$, the adaptive law of switching control is updated below:

$$
\dot{\widehat{E}}_{4}=\rho_{4}\left|S_{4}\right| \text {. }
$$

Then, 


$$
\begin{aligned}
\dot{\Gamma}_{4} & =-\widehat{E}_{4}\left|S_{4}\right|-\varepsilon_{4} S_{4}+\frac{1}{\rho_{4}}\left(\widehat{E}_{4}-E_{4}\right) \dot{\widehat{E}}_{4} \\
& \leq-E_{4}\left|S_{4}\right|-\varepsilon_{4} S_{4} \\
& \leq-\left(E_{4}-\left|\varepsilon_{4}\right|\right)\left|S_{4}\right| \\
& \leq 0
\end{aligned}
$$

The similar design processes are presented to design the trajectory tracking of pitch $x_{9}$ and yaw $x_{11}$ using the dynamic surface integral sliding mode control. The integral sliding mode manifolds (T2.10), (T2.16) and the first-order filter (T2.9), (T2.15) are chosen properly. The adaptive law and the switching control (T2.11), (T2.18) of the pitch and yaw are presented in Table 2. And (T2.12), (T2.18) are the actual control inputs of the pitch and yaw equation. $c_{5}, c_{6}, \tau_{5}$, $\tau_{6}, k_{9}, k_{10}, k_{11}, k_{12}, \eta_{5}, \eta_{6}, \rho_{5}$, and $\rho_{6}$ are positive constants which need to be assigned to meet the performance requirements of the pitch and yaw angle system. The derivatives of Lyapunov function of pitch and yaw angle are presented as follows:

$$
\begin{aligned}
\dot{\Gamma}_{5}= & -\widehat{E}_{5}\left|S_{5}\right|-\varepsilon_{5} S_{5}+\frac{1}{\rho_{5}}\left(\widehat{E}_{5}-E_{5}\right) \dot{\widehat{E}}_{5} \\
& \leq-E_{5}\left|S_{5}\right|-\varepsilon_{5} S_{5} \\
& \leq-\left(E_{5}-\left|\varepsilon_{5}\right|\right)\left|S_{5}\right| \\
& \leq 0 \\
\dot{\Gamma}_{6} & =-\widehat{E}_{6}\left|S_{6}\right|-\varepsilon_{6} S_{6}+\frac{1}{\rho_{6}}\left(\widehat{E}_{6}-E_{6}\right) \dot{\widehat{E}}_{6} \\
& \leq-E_{6}\left|S_{6}\right|-\varepsilon_{6} S_{6} \\
& \leq-\left(E_{6}-\left|\varepsilon_{6}\right|\right)\left|S_{6}\right| \\
& \leq 0 .
\end{aligned}
$$

\section{B. The Proof of Theorem}

Proof. Consider the Lyapunov function candidate

$$
\begin{aligned}
V & =V_{1}+V_{2}, \\
V_{1} & =\frac{1}{2} \sum_{i=1}^{6}\left(e_{2 i-1}^{2}+y_{2 i}^{2}\right), \\
V_{2} & =\frac{1}{2} \sum_{i=1}^{6}\left(S_{i}^{2}+\frac{1}{\eta_{i}} \widetilde{\alpha}_{i}^{T} \widetilde{\alpha}_{i}+\frac{1}{\rho_{i}} \widetilde{E}_{i}^{2}\right) .
\end{aligned}
$$

The derivative of $V_{1}$ with respect to time can be obtained as follows:

$$
\dot{V}_{1}=\sum_{i=1}^{6}\left(e_{2 i-1} \dot{e}_{2 i-1}+y_{2 i} \dot{y}_{2 i}\right)
$$

Substitute the adaptive law and the switching control law into (B.2):

$$
\begin{aligned}
\dot{V}_{1} & =\sum_{i=1}^{6}\left(-c_{i} e_{2 i-1}^{2}-\frac{y_{2 i}^{2}}{\tau_{i}}+\left|y_{2 i} B_{2 i}\right|\right) \\
& \leq \sum_{i=1}^{6}\left(-c_{i} e_{2 i-1}^{2}-\frac{y_{2 i}^{2}}{\tau_{i}}+\left|y_{2 i} B_{2 i}\right|\right) .
\end{aligned}
$$

Noting that, for any positive number $\lambda,\left|y_{2 i} B_{2 i}\right| \leq\left(y_{2 i}^{2} B_{2 i}^{2} / 2 \lambda\right)+(\lambda / 2)$. Assume that $\left|B_{2 i}\right|<M_{2 i}$, where $M_{2 i}$ is a positive constant.

$$
\begin{aligned}
\dot{V}_{1} & \leq \sum_{i=1}^{6}\left(-c_{i} e_{2 i-1}^{2}-\frac{y_{2 i}^{2}}{\tau_{i}}+\frac{y_{2 i}^{2} B_{2 i}^{2}}{2 \lambda}+\frac{\lambda}{2}\right) \\
& \leq \sum_{i=1}^{6}\left(-c_{i} e_{2 i-1}^{2}-\left(\frac{2}{\tau_{i}}-\frac{M_{2 i}^{2}}{\lambda}\right) \frac{y_{2 i}^{2}}{2}+\frac{\lambda}{2}\right) .
\end{aligned}
$$

Let

$$
\left\{\begin{array}{l}
c_{i}=\alpha_{0}, \\
\frac{2}{\tau_{i}}-\frac{M_{2 i}^{2}}{\lambda}=\alpha_{0}
\end{array}\right.
$$

where $\alpha_{0}$ is a positive constant.

$$
\dot{V}_{1} \leq-\alpha_{0} V_{1}+3 \lambda
$$

Let $\alpha_{0}>(3 \lambda / 2 p)$; then $\dot{V}_{1} \leq 0$ on $V_{1}=p$. And $V_{1} \leq p$ is an invariant set. Thus, if $V_{1}(0) \leq p$, then $V_{1}(t) \leq p$ for all $t \geq 0$. Thus, (B.6) holds for all $V_{1}(t) \leq p$ and all $t \geq 0$.

$$
0 \leq V_{1}(t) \leq \frac{3 \lambda}{4 \alpha_{0}}+\left(V_{1}(0)-\frac{3 \lambda}{4 \alpha_{0}}\right) e^{-2 \alpha_{0} t}, \quad \forall t \geq 0 .
$$

From (B.7), we can get that $V_{1}(t)$ is eventually is bounded by $\left(3 \lambda / 4 \alpha_{0}\right)$.

$$
V_{2}=\frac{1}{2} \sum_{i=1}^{6}\left(S_{i}^{2}+\frac{1}{\eta_{i}} \widetilde{\alpha}_{i}^{T} \widetilde{\alpha}_{i}+\frac{1}{\rho_{i}} \widetilde{E}_{i}^{2}\right) .
$$

The derivative of $V_{2}$ with respect to time can be obtained as follows:

$$
\begin{aligned}
\dot{V}_{2} & =\sum_{i=1}^{6}\left(S_{i} \dot{S}_{i}+\frac{1}{\eta_{i}} \widetilde{\alpha}_{i}^{T} \dot{\tilde{\alpha}}_{i}+\frac{1}{\rho_{i}} \widetilde{E}_{i} \dot{\widetilde{E}}_{i}\right) \\
& =\sum_{i=1}^{6}\left[S_{i}\left(U_{i f z}+U_{i v s}-U_{i}^{*}\right)+\frac{1}{\eta_{i}} \widetilde{\alpha}_{i}^{T} \dot{\tilde{\alpha}}_{i}+\frac{1}{\rho_{i}} \widetilde{E}_{i} \dot{\widetilde{E}}_{i}\right] \\
& =\sum_{i=1}^{6}\left[\widetilde{\alpha}_{i}^{T}\left(S_{i} \xi_{i}+\frac{1}{\eta_{i}} \dot{\widetilde{\alpha}}_{i}\right)+S_{i}\left(U_{i v s}-\varepsilon_{i}\right)+\frac{1}{\rho_{i}} \widetilde{E}_{i} \dot{\widetilde{E}}_{i}\right] .
\end{aligned}
$$

Substitute the adaptive laws and the switching control laws (T1.5), (T1.11), (T1.17), (T2.5), (T2.11), and (T2.17) into (B.9) 


$$
\begin{aligned}
\dot{V}_{2} & =\sum_{i=1}^{6}\left[-\widehat{E}_{i}\left|S_{i}\right|-\varepsilon_{i} S_{i}+\left(\widehat{E}_{i}-E_{i}\right)\left|S_{i}\right|\right] \\
& =\sum_{i=1}^{3}\left[-E_{i}\left|S_{i}\right|-\varepsilon_{i} S_{i}\right] \\
& \leq \sum_{i=1}^{3}\left[-\left(E_{i}-\left|\varepsilon_{i}\right|\right)\left|S_{i}\right|\right] \\
& \leq 0
\end{aligned}
$$

where $E_{i} \geq\left|\varepsilon_{i}\right|$, so we can know that $V_{2}(t)$ is eventually is bounded. Hence, all signals in the closed loop are ultimately bounded. Particularly, the tracking errors and the estimation errors can be arbitrarily small.

\section{Data Availability}

The data used to support the findings of this study are available from the corresponding author upon request.

\section{Conflicts of Interest}

The authors declare that there are no conflicts of interest regarding the publication of this paper.

\section{Acknowledgments}

This work was supported in part by the NSF of China under Grant no. 61673101 and the Thirteenth Five-Year Science Research Plan of Jilin Province under Grant JJKH20200119KJ.

\section{References}

[1] B. Zhao, B. Xian, Y. Zhang, and X. Zhang, "Nonlinear robust adaptive tracking control of a quadrotor uav via immersion and invariance methodology," IEEE Transactions on Industrial Electronics, vol. 62, no. 5, pp. 2891-2902, 2014.

[2] R. Mahony, V. Kumar, and P. Corke, "Multirotor aerial vehicles: modeling, estimation, and control of quadrotor," IEEE Robotics \& Automation Magazine, vol. 19, no. 3, pp. 20-32, 2012.

[3] T. Huang, D. Huang, Z. Wang, and A. Shah, "Robust tracking control of a quadrotor UAV based on adaptive sliding mode controller," Complexity, vol. 2019, Article ID 7931632, 15 pages, 2019.

[4] L. Besnard, Y. B. Shtessel, and B. Landrum, "Quadrotor vehicle control via sliding mode controller driven by sliding mode disturbance observer," Journal of the Franklin Institute, vol. 349, no. 2, pp. 658-684, 2012.

[5] Z. T. Dydek, A. M. Annaswamy, and E. Lavretsky, "Adaptive control of quadrotor uavs: a design trade study with flight evaluations," IEEE Transactions on Control Systems Technology, vol. 21, no. 4, pp. 1400-1406, 2012.

[6] X. Zhang, Y. Wang, G. Zhu et al., "Compound adaptive fuzzy quantized control for quadrotor and its experimental verification," IEEE Transactions on Cybernetics, pp. 1-13, 2020.

[7] X. Dong, B. Yu, Z. Shi, and Y. Zhong, "Time-varying formation control for unmanned aerial vehicles: theories and applications," IEEE Transactions on Control Systems Technology, vol. 23, no. 1, pp. 340-348, 2014.
[8] J.-J. Xiong and E.-H. Zheng, "Position and attitude tracking control for a quadrotor uav," ISA Transactions, vol. 53, no. 3, pp. 725-731, 2014.

[9] P. E. Pounds, D. R. Bersak, and A. M. Dollar, "Stability of small-scale uav helicopters and quadrotors with added payload mass under pid control," Autonomous Robots, vol. 33, no. 1-2, pp. 129-142, 2012.

[10] Z. Zuo, "Trajectory tracking control design with commandfiltered compensation for a quadrotor," IET Control Theory \& Applications, vol. 4, no. 11, pp. 2343-2355, 2010.

[11] S. He, K. Relf, and R. Unbehauen, "A neural approach for control of nonlinear systems with feedback linearization," IEEE Transactions on Neural Networks, vol. 9, no. 6, pp. 1409-1421, 1998.

[12] I. A. Raptis, K. P. Valavanis, and W. A. Moreno, “A novel nonlinear backstepping controller design for helicopters using the rotation matrix," IEEE Transactions on Control Systems Technology, vol. 19, no. 2, pp. 465-473, 2010.

[13] G. Zhu and J. Liu, "Neural network-based adaptive backstepping control for hypersonic flight vehicles with prescribed tracking performance," Mathematical Problems in Engineering, vol. 2015, Article ID 591789, 10 pages, 2015.

[14] Y. Yu, C. Zhang, and M. Zhou, "Narmax model-based hysteresis modeling of magnetic shape memory alloy actuators," IEEE Transactions on Nanotechnology, vol. 19, pp. 1-4, 2020.

[15] X. Zhang, Y. Wang, X. Chen et al., "Decentralized adaptive neural approximated inverse control for a class of large-scale nonlinear hysteretic systems with time delays," IEEE Transactions on Systems, Man, and Cybernetics: Systems, vol. 49, no. 12, pp. 2424-2437, 2019.

[16] Z. Jia, J. Yu, Y. Mei, Y. Chen, Y. Shen, and X. Ai, "Integral backstepping sliding mode control for quadrotor helicopter under external uncertain disturbances," Aerospace Science and Technology, vol. 68, pp. 299-307, 2017.

[17] Y. Kui, G. Feng, Y. Liying, H. Yuqing, and H. Jianda, "Sliding Mode Control for a Quadrotor Slung Load System," in Proceedings of the 2017 36th Chinese Control Conference (CCC), pp. 3697-3703, IEEE, Dalian, China, July 2017.

[18] Y. Zou, "Nonlinear robust adaptive hierarchical sliding mode control approach for quadrotors," International Journal of Robust and Nonlinear Control, vol. 27, no. 6, pp. 925-941, 2017.

[19] X. Xiang, C. Liu, H. Su, and Q. Zhang, "On decentralized adaptive full-order sliding mode control of multiple uavs," ISA Transactions, vol. 71, pp. 196-205, 2017.

[20] T. Li, Y. Zhang, and B. W. Gordon, "Nonlinear fault-tolerant control of a quadrotor uav based on sliding mode control technique," IFAC Proceedings Volumes, vol. 45, no. 20, pp. 1317-1322, 2012.

[21] B. Sumantri, N. Uchiyama, and S. Sano, "Least square based sliding mode control for a quad-rotor helicopter and energy saving by chattering reduction," Mechanical Systems and Signal Processing, vol. 66-67, pp. 769-784, 2016.

[22] B. Xu, X. Liu, H. Wang, and Y. Zhou, "Event-triggered adaptive backstepping control for strict-feedback nonlinear systems with zero dynamics," Complexity, vol. 2019, Article ID 7890968, 13 pages, 2019.

[23] S. Zhang and C. Chen, "Backstepping based nonlinear integral sliding mode control for quadrotors under external disturbances," in Proceedings of the 2019 Chinese Control Conference (CCC), pp. 8355-8359, IEEE, Guangzhou, China, July 2019.

[24] M. A. M. Basri, "Robust backstepping controller design with a fuzzy compensator for autonomous hovering quadrotor uav," 
Iranian Journal of Science and Technology, Transactions of Electrical Engineering, vol. 42, no. 3, pp. 379-391, 2018.

[25] H. Shraim, Y. Harkouss, and H. Bazzi, "A novel hybrid backstepping and fuzzy logic control strategy for a quadcopter," The Aeronautical Journal, vol. 121, no. 1244, pp. 1444-1463, 2017.

[26] X. Zhang, X. Chen, G. Zhu, and C.-Y. Su, “Output feedback adaptive motion control and its experimental verification for time-delay nonlinear systems with asymmetric hysteresis," IEEE Transactions on Industrial Electronics, vol. 67, no. 8, pp. 6824-6834, 2020.

[27] X. Tang, "Adaptive neural network dynamic surface control for a class of unknow time-delay nonlinear hysteresis system," Journal of Northeast Electric Power University, vol. 38, no. 3, pp. 52-60, 2018.

[28] Z. Li, J. Shan, and U. Gabbert, "Dynamics modeling and inversion-based synchronized model predictive control for a Fabry-perot spectrometer," IEEE/ASME Transactions on Mechatronics, vol. 24, no. 4, pp. 1818-1828, 2019.

[29] G. Zhu, L. Nie, M. Zhou, X. Zhang, L. Sun, and C. Zhong, "Adaptive fuzzy dynamic surface control for multi-machine power system based on composite learning method and disturbance observer," IEEE Access, vol. 8, pp. 163163-163175, 2020.

[30] X. Zhang, B. Li, X. Chen, Z. Li, Y. Peng, and C.-Y. Su, "Adaptive implicit inverse control for a class of discrete-time hysteretic nonlinear systems and its application," IEEE/ASME Transactions on Mechatronics, vol. 25, no. 4, pp. 2112-2122, 2020.

[31] C. Yang, Y. Jiang, W. He, J. Na, Z. Li, and B. Xu, “Adaptive parameter estimation and control design for robot manipulators with finite-time convergence," IEEE Transactions on Industrial Electronics, vol. 65, no. 10, pp. 8112-8123, 2018.

[32] Z. Li and J. Shan, "Inverse compensation based synchronization control of the piezo-actuated Fabry-perot spectrometer," IEEE Transactions on Industrial Electronics, vol. 64, no. 11, pp. 8588-8597, 2017.

[33] J. Yu, Y. Ma, H. Yu, and C. Lin, "Adaptive fuzzy dynamic surface control for induction motors with iron losses in electric vehicle drive systems via backstepping," Information Sciences, vol. 376, pp. 172-189, 2017.

[34] Y. Jiang, C. Yang, S.-1. Dai, and B. Ren, "Deterministic learning enhanced neutral network control of unmanned helicopter," International Journal of Advanced Robotic Systems, vol. 13, no. 6, 2016.

[35] X. Shao, J. Liu, H. Cao, C. Shen, and H. Wang, "Robust dynamic surface trajectory tracking control for a quadrotor uav via extended state observer," International Journal of Robust and Nonlinear Control, vol. 28, no. 7, pp. 2700-2719, 2018.

[36] B. Xu, C. Yang, and Y. Pan, "Global neural dynamic surface tracking control of strict-feedback systems with application to hypersonic flight vehicle," IEEE Transactions on Neural Networks and Learning Systems, vol. 26, no. 10, pp. 25632575, 2015.

[37] X. Zhang, Y. Wang, C. Wang, C.-Y. Su, Z. Li, and X. Chen, "Adaptive estimated inverse output-feedback quantized control for piezoelectric positioning stage," IEEE Transactions on Cybernetics, vol. 49, no. 6, pp. 2106-2118, 2019.

[38] C. Yang, Y. Jiang, J. Na, Z. Li, L. Cheng, and C.-Y. Su, "Finitetime convergence adaptive fuzzy control for dual-arm robot with unknown kinematics and dynamics," IEEE Transactions on Fuzzy Systems, vol. 27, no. 3, pp. 574-588, 2018.
[39] D. Huang, C. Yang, Y. Pan, and L. Cheng, "Composite learning enhanced neural control for robot manipulator with output error constraints," IEEE Transactions on Industrial Informatics, vol. 17, no. 1, pp. 209-218, 2019.

[40] G. Zhu, L. Nie, Z. Lv, L. Sun, X. Zhang, and C. Wang, "Adaptive fuzzy dynamic surface sliding mode control of large-scale power systems with prescribe output tracking performance," ISA Transactions, vol. 99, pp. 305-321, 2020.

[41] S. Mobayen, F. Tchier, and L. Ragoub, "Design of an adaptive tracker for n-link rigid robotic manipulators based on supertwisting global nonlinear sliding mode control," International Journal of Systems Science, vol. 48, no. 9, pp. 1990-2002, 2017.

[42] S. He and J. Song, "Finite-time sliding mode control design for a class of uncertain conic nonlinear systems," IEEE/CAA Journal of Automatica Sinica, vol. 4, no. 4, pp. 809-816, 2017.

[43] G. Zhu, S. Wang, L. Sun, W. Ge, and X. Zhang, "Output feedback adaptive dynamic surface sliding mode control for quadrotor uavs with tracking error constraints," Complexity, vol. 2020, Article ID 8537198, 23 pages, 2020.

[44] M. Manceur, N. Essounbouli, and A. Hamzaoui, "Secondorder sliding fuzzy interval type-2 control for an uncertain system with real application," IEEE Transactions on Fuzzy Systems, vol. 20, no. 2, pp. 262-275, 2011.

[45] S. Mobayen, "Design of cnf-based nonlinear integral sliding surface for matched uncertain linear systems with multiple state-delays," Nonlinear Dynamics, vol. 77, no. 3, pp. 1047-1054, 2014.

[46] M. Golestani, F. Tchier, and S. Mobayen, "Adaptive finitetime tracking control of uncertain non-linear n-order systems with unmatched uncertainties," IET Control Theory \& Applications, vol. 10, no. 14, pp. 1675-1683, 2016.

[47] I. González, S. Salazar, and R. Lozano, "Chattering-free sliding mode altitude control for a quadrotor aircraft: real-time application," Journal of Intelligent \& Robotic Systems, vol. 73, no. 1-4, pp. 137-155, 2014.

[48] S. Mobayen, "A novel global sliding mode control based on exponential reaching law for a class of under actuated systems with external disturbances," Journal of Computational and Nonlinear Dynamics, vol. 11, no. 2, 9 pages, Article ID 021011, 2016.

[49] M. Chen, Q.-X. Wu, and R.-X. Cui, "Terminal sliding mode tracking control for a class of siso uncertain nonlinear systems," ISA Transactions, vol. 52, no. 2, pp. 198-206, 2013.

[50] E.-H. Zheng, J.-J. Xiong, and J.-L. Luo, "Second order sliding mode control for a quadrotor uav," ISA Transactions, vol. 53, no. 4, pp. 1350-1356, 2014.

[51] F. Chen, R. Jiang, K. Zhang, B. Jiang, and G. Tao, "Robust backstepping sliding-mode control and observer-based fault estimation for a quadrotor UAV," IEEE Transactions on Industrial Electronics, vol. 63, no. 8, pp. 5044-5056, 2016. 